ERRATA

\title{
Erratum to: Society of General Internal Medicine 34th Annual Meeting, Phoenix, Arizona, May 4-7, 2011
}

J Gen Intern Med 26(10):1219-34

DOI: $10.1007 / \mathrm{s} 11606-011-1814-6$

( ) Society of General Internal Medicine 2011

$\mathrm{T}$

he below text was omitted from the original publication.

\section{ABSTRACTS}

A Telephone-based Lifestyle Intervention to Lower Risk Factors in Ethnic Minority and Lower Socioeconomic Status Adults at Risk of Diabetes Alka M. Kanaya ${ }^{1}$; Jasmine Santoyo-Olsson ${ }^{2}$; Steven Gregorich ${ }^{2}$; Melanie Grossman ${ }^{2}$; Tanya Moore ${ }^{3}$; Anita Stewart ${ }^{2}$. ${ }^{1}$ University of California, San Francisco, San Francisco, California ; ${ }^{2}$ UCSF, San Francisco, California ; ${ }^{3}$ Berkeley Dept of Public Health, Berkeley, California . (Tracking ID \# 10230)

BACKGROUND: Intensive lifestyle interventions are effective in lowering diabetes incidence in randomized clinical trials, but few community translations exist using less intensive interventions. We determined the effect of a translational lifestyle program (Live Well, Be Well) designed to reduce diabetes risk factors in lower-socioeconomic status ethnic minority groups.

METHODS: We conducted a randomized controlled trial with a wait-list control group in a Northern California community. Individuals age â\%o $¥ 25$ years at risk for diabetes by self-report risk assessment and elevated fasting fingerstick glucose were eligible. The intervention group received individualized lifestyle counseling. Public health department staff provided education and skills training via 2 in-person sessions, followed by goal-focused telephone counseling. Primary outcomes included fasting glucose, triglycerides, HDL-, LDL-cholesterol, weight, waist circumference, and systolic blood pressure at 6 and 12 months. Secondary outcomes included diet, physical activity, and health-related quality of life.

RESULTS: Of 230 participants randomized, 113 (49\%) were assigned to the lifestyle intervention. Significant group differences in 6-month change scores were observed for weight and triglycerides. The intervention group lost approximately 2 pounds more than the control group $(\mathrm{P}=.03)$ and had greater reductions in triglycerides (difference $23 \mathrm{mg} / \mathrm{dL}, \mathrm{P}=.02$ ). The intervention group consumed approximately 7.1 fewer grams of fat per day $(\mathrm{P}=.04)$ and consumed more fruits and vegetables $(\mathrm{P}=.01)$ than the control group. Overall study retention was $92 \%$.

The online version of the original article can be found at http://dx.doi. org/10.1007/s11606-011-1730-9.

Published online August 24, 2011
CONCLUSION: Despite challenges designing effective community-based translations for lower SES communities, this individually-tailored, low intensity lifestyle program was associated with improvements in some diabetes risk factors. LWBW provides a promising model for reducing disparities in diabetes risk and an alternative to group-based community translations.

Adiponectin and All-Cause Mortality in a Cohort of Elderly People with Type 2 Diabetes Jessica Rohman Singer ${ }^{1}$; Walter Palmas ${ }^{1}$; Steven Shea ${ }^{1}$; Jose Alejandro Luchsinger ${ }^{1} .{ }^{1}$ Division of General Medicine, Department of Medicine, Columbia University College of Physicians and Surgeons, New York, New York . (Tracking ID \# 7871)

BACKGROUND: Nearly a quarter of Americans over the age of 60 are estimated to have type 2 diabetes. The Action to Control Cardiovascular Risk in Diabetes Trial demonstrated that tight glycemic control was associated with increased mortality, while tight lipid and blood pressure control had no association with mortality. These surprising findings raise the possibility of novel predictors of mortality in people with type 2 diabetes. We sought to explore whether insulin resistance measured by adiponectin level was related to increased mortality in people with type 2 diabetes. High levels of adiponectin are known to be associated with lower risk of developing type 2 diabetes, but no data have been published to our knowledge regarding the association of adiponectin level and mortality specifically in patients with type 2 diabetes. Data regarding adiponectin level and mortality in non-diabetic populations are inconsistent.

METHODS: Participants were a subsample of 627 subjects from the Informatics for Diabetes Education and Telemedicine project (IDEATel) who were enrolled in a substudy. IDEATel was a CMS supported randomized controlled trial designed to evaluate the effectiveness of telemedicine case management in elderly Medicare beneficiaries with type 2 diabetes but without severe co-morbid disease. Subjects were enrolled in IDEATel from December 2000 to October 2005. Adiponectin was measured on frozen serum samples for all subjects and mortality data were collected from the National Death Index through 11/30/2009. ANOVA and Chi-square were used to compare relevant continuous and categorical variables across quartiles of adiponectin. Cox proportional hazards regression was performed to examine the relationship between adiponectin level and allcause mortality adjusting for age, gender, race/ethnicity, hemoglobin Alc, blood pressure, LDL, HDL, triglycerides, BMI, albumin/creatinine ratio, creactive protein, active tobacco use and medication usage, specifically thiazolidinediones (TZDs).

RESULTS: Subjects had a mean age of 72 , were $70 \%$ women, $83 \%$ Hispanic and 15\% African American. Compared to the lowest adiponectin quartile, those in the highest adiponectin quartile had higher HDL (51.4 +/- 16.7 vs. $42.7+/-12.3$; $\mathrm{p}<0.001)$, higher log albumin/ creatinine $(1.6+/-0.6$ vs $1.3+/-0.5 ; \mathrm{p}=0.0037)$, higher frequency of 
TZD use (58.6\% vs. 5.8\%; $\mathrm{p}<0.0001)$, lower triglycerides $(121.6+/-61.3$ vs $144.6+/-77.9 ; \mathrm{p}=0.005)$ and lower CRP $(0.3+/-1.3$ vs. $1.0+/-1.3$; $\mathrm{p}<0.0001)$. Cox regression models demonstrated that compared with those in the lowest quartile of adiponectin, those in the highest adiponectin quartile had an increased hazard of death with a fully adjusted hazard ratio of $4.0(95 \% \mathrm{CI} 1.7,9.2)$; p-value $=0.0003$ for trend across quartiles of adiponectin level (Table 1). These results remained consistent when the analysis was stratified by age, gender and TZD usage and after excluding those subjects who died within one year of adiponectin sampling. We conducted a secondary analysis in a sample of 464 participants for whom we were able to calculate change in weight predating the adiponectin measurement. After adjustment for weight gain or loss of more than $10 \%$ of body weight, the association between adiponectin and higher mortality remained significant with a fully adjusted hazard ratio of $3.1(95 \%$ CI $1.2,7.9) ; \mathrm{p}=0.01$ for trend across quartiles of adiponectin level.

CONCLUSION: Higher adiponectin level was associated with higher allcause mortality in a cohort of elderly people with type 2 diabetes.

An International, Randomized Non-Inferiority Trial of Outpatient Versus Inpatient Treatment for Pulmonary Embolism Drahomir $\underline{\text { Aujesky }}{ }^{1}$; Pierre-Marie Roy ${ }^{2}$; Franck Verschuren ${ }^{3}$; Marc Righini ${ }^{4}$; Joseph Osterwalder ${ }^{5}$; Michael Egloff ${ }^{6}$; Bertrand Renaud ${ }^{7}$; Peter Verhamme ${ }^{8}$; Catherine Legall ${ }^{9}$; Olivier Sanchez ${ }^{10}$; Roslyn A. Stone ${ }^{11}$; Nathan Pugh ${ }^{12}$; Alfred Ngako ${ }^{7}$; Jacques Cornuz ${ }^{13}$; Olivier Hugli ${ }^{14}$; Hans-Juerg Beer ${ }^{6}$; Arnaud Perrier ${ }^{4}$; Michael J. Fine ${ }^{11}$; Donald M. Yealy ${ }^{12}$. ${ }^{1}$ Bern University Hospital, Bern, N/A ; ${ }^{2}$ University of Angers, Angers, N/A ; ${ }^{3}$ University of Louvain, Louvain, N/A ; ${ }^{4}$ University of Geneva, Geneva, N/A ; ${ }^{5}$ Cantonal Hospital of St. Gallen, St. Gallen, N/A ; ${ }^{6}$ Cantonal Hospital of Baden, Baden, N/A ; ${ }^{7}$ Creteil University Hospital, Creteil, N/A ; ${ }^{8}$ University of Leuven, Leuven, N/A ; ${ }^{9}$ University of Argenteuil, Argenteuil, N/A ; ${ }^{10}$ Hopital Europeen Georges Pompidou, Paris, N/A ; ${ }^{11}$ University of Pittsburgh and VA Pittsburgh Healthcare System, Pittsburgh, Pennsylvania ; ${ }^{12}$ University of Pittsburgh, Pittsburgh, Pennsylvania ; ${ }^{13}$ University of Lausanne, Lausanne, N/A . (Tracking ID \# 10107)

BACKGROUND: Although medical specialty practice guidelines recommend outpatient treatment for selected, hemodynamically stable patients with pulmonary embolism (PE), the outcomes of outpatient management remain unknown. Our aim was to compare the effectiveness and safety of managing low-risk patients with $\mathrm{PE}$ in the outpatient versus inpatient setting.

METHODS: We conducted an international, randomized non-inferiority trial at 19 emergency departments (EDs) in Europe and the United States. We randomly assigned 344 consecutive patients (02/2007-06/2010) with acute, symptomatic, objectively confirmed PE who were at low risk of death (PE Severity Index risk classes I and II) to initial outpatient versus inpatient treatment with subcutaneous enoxaparin for at least 5 days, followed by oral anticoagulation for at least 90 days. Patients assigned to outpatient treatment received standardized teaching on self-injection with enoxaparin and were discharged from the ED within 24 hours of randomization. Patients assigned to receive inpatient treatment were admitted to the hospital, with their readiness for hospital discharge autonomously determined by their treating physicians. The primary outcome was symptomatic, objectively confirmed, recurrent venous thromboembolism (VTE) within 90 days; secondary outcomes were 90-day mortality, major bleeding at 14 and 90 days, and medical resource utilization (i.e., hospital bed-days). We compared the percentages of outpatients and inpatients that experienced recurrent VTE, death, and major bleeding. Non-inferiority was defined a priori as an exact upper 95\% confidence interval (CI) limit of less than $4 \%$ for the difference. We analyzed data according to the intention-totreat principle.

RESULTS: Overall, 1 (0.6\%) of 171 patients randomized to outpatient care and 0 of 168 patients randomized to inpatient care developed recurrent VTE within 90 days $(\mathrm{P}=0.001$; $95 \%$ upper $\mathrm{CI}$ limit $=2.7 \%)$. Only $1(0.6 \%)$ patient in each treatment arm died within 90 days $(\mathrm{P}=0.005 ; 95 \%$ upper $\mathrm{CI}$ limit $=2.1 \%)$. Within 14 days, $2(1.2 \%)$ outpatients and 0 inpatients had major bleeding ( $\mathrm{P}=0.001$; 95\% upper $\mathrm{CI}$ limit $=3.6 \%)$. Collectively, these results support non-inferiority for recurrent VTE, mortality, and major bleeding. Within 90 days, 1 additional outpatient developed major bleeding, totaling 3 (1.8\%) outpatients and 0 inpatients with this outcome $(\mathrm{P}=0.086$; $95 \%$ upper CI limit=4.5\%); although the $95 \%$ upper CI limit slightly exceeds our non-inferiority threshold, this third bleeding episode occurred on day 50 and was not temporally related to the initial use of enoxaparin or randomization to outpatient treatment. Outpatients had a significantly shorter length of stay than inpatients ( 0.5 vs 3.9 days; $\mathrm{P}<0.001)$.

CONCLUSION: In low-risk patients defined using the PE Severity Index, outpatient treatment with low-molecular-weight heparin is feasible, is not less effective or safe than inpatient treatment, and provides a substantial reduction in health care resource use.

Blood Pressure among Older U.S. Adults with Diabetes Differs by Social Support and Income Laura Plantinga ${ }^{1}$; Urmimala Sarkar ${ }^{1}$; Dean Schillinger ${ }^{1}$. ${ }^{1}$ Center for Vulnerable Populations, San Francisco General Hospital, and University of California, San Francisco, California . (Tracking ID \# 10590)

BACKGROUND: Persons with diabetes commonly have hypertension, which substantially increases the risk of macro- and microvascular disease. Social support has been shown to be associated with improved blood pressure control. Having emotional support might allow greater adherence to medications and dietary and activity recommendations and decrease overall stress, leading to greater blood pressure control. However, a person's financial resources could modify any such effect. Whether the association of social support with blood pressure control differs by income among older persons with diabetes mellitus has not been explored.

METHODS: We used the National Health and Nutrition Examination Survey (NHANES) 1999-2008 to examine associations of social support with blood pressure control among 1,681 U.S. adults ( $\geq 60$ years old) with self-reported diabetes and information on social support. Standardized questionnaires assessed, over the previous 12 months, whether the respondent had emotional support ["Can you count on anyone to provide you with emotional support such as talking over problems or helping you make a difficult decision?" (yes/no)] and total number of respondent's close friends. Blood pressure was measured (up to four measurements) by standardized protocol and the average mean arterial pressure (MAP) was calculated for each participant. Multivariable logistic regression with U.S. population-based weighting was used to obtain adjusted estimates.

RESULTS: Overall, $92.4 \%$ reported having someone who provided emotional support and the mean number of close friends was 7.7. After adjustment for age, gender, race/ethnicity, time since diabetes diagnosis, and last visit to a doctor ( $\leq 1$ vs. $>1$ year), reported emotional support was associated with higher MAP in those with lower income and with lower MAP in those with higher income (see below). A similar pattern was seen for social network, with a greater reported number of close friends being associated with lower MAP among those with higher income. The effect modifications observed for blood pressure and emotional support by income were strongest in persons with diabetes who were male, white, and/or reported no outside financial support.

CONCLUSION: Older persons with diabetes in the United States have relatively high levels of social support overall. Having a larger and more 
supportive network appeared to be beneficial for blood pressure control among those with higher income. The observed association of worse blood pressure control among lower-income individuals with greater emotional support, particularly among those without outside financial support, suggests that the emotional support received by those with fewer resources may increase stress (e.g., inability to act upon health advice due to lack of access) and/or may lead to health behaviors that worsen hypertension (e.g., poor dietary choices in social situations). Those with fewer resources may require greater, more material support to control their blood pressure. Further study of the mechanisms by which social support influences blood pressure control among poor elders with diabetes is needed.

Characteristics and Predictors of Low-income Black Patients' Discussions about Pain During Primary Care Interactions Stephen G Henry $^{1}$; Louis A. Penner ${ }^{2}$; Susan Eggly ${ }^{2} .{ }^{1}$ VA Ann Arbor Health Care System, Ann Arbor, Michigan ; ${ }^{2}$ Karmanos Cancer Institute / Wayne State University, Detroit, Michigan . (Tracking ID \# 10941)

BACKGROUND: Difficulties in communication about pain during primary care interactions is a potential contributor to racial disparities in pain management. Information about how primary care physicians and vulnerable minority populations discuss pain is important for reducing these disparities. Study objectives are to describe the frequency, characteristics, and factors that predict the presence and duration of discussions about pain during primary care interactions in a low-income black population.

METHODS: Developed coding scheme for assessing characteristics and length of pain-related discussions from existing video-recorded primary care interactions involving 133 black, low-income patients and 17 primary care physicians. Multiple logistic and linear regressions to evaluate patient and visit characteristics associated with pain discussions. Measures included patientsâ€тM baseline pain severity and health status measured using the MOS 20-item health survey; fraction of the total interaction spent discussing pain; descriptive characteristics of pain discussions; the presence or absence of continuity of care.

RESULTS: $69 \%$ of interactions included pain discussions lasting a median of 5.4 minutes (IQR 2.1, 8.4) and constituting 30\% of total interaction time (IQR 14\%, 52\%). Worse pain severity (adjusted OR 0.61, 95\% CI[0.40,0.93] and physical function (adjusted OR 0.63, 95\% CI [0.45,0.89]) were significantly associated with the presence of pain discussions. Worse pain (coefficient $-4.51,95 \% \mathrm{CI}[-8.82,-0.20]$ ), better physical function (coefficient 3.20, 95\%CI[0.23,5.88]), and absent continuity of care (coefficient $-14.04,95 \% \mathrm{CI}[-23.60,-4.47$ ] were significantly associated with a higher fraction of the total interaction spent discussing pain. Pain severity was only related to time spent discussing pain when continuity was absent.

CONCLUSION: Pain is a frequent and significant topic during primary care interactions involving low-income black patients. Continuity of care, pain severity, and physical function are significantly associated with these discussions. Direct observation can provide important data for evaluating the content and quality of pain management in primary care.

Changing from Guaiac Fecal Occult Blood Testing (gFOBT) to Fecal Immunochemical Testing (FIT) Increases Patient Completion of Colon Cancer Screening David Mosen ${ }^{1}$; Elizabeth G Liles ${ }^{1}$; Adrianne C Feldstein ${ }^{2}$; Nancy Perrin ${ }^{1}$; Ana G Rosales ${ }^{1}$; David $\mathrm{H}$ Smith $^{3}$. ${ }^{1}$ Kaiser Permanente Northwest, Portland, Oregon ; ${ }^{2}$ Kaiser Permanente, Portland, Oregon . (Tracking ID \# 10707)

BACKGROUND: Colorectal cancer (CRC) is the second leading cause of cancer death in the United States. Timely CRC screening can reduce CRC-related mortality, yet screening rates remain low. The fecal immunochemical test (FIT) and high-sensitivity guaiac fecal occult blood test (gFOBT) are fecal tests currently considered valid CRC screening options. The single-sample OC-Micro FIT is more sensitive at detecting both $\mathrm{CRC}$ and precancerous adenomas than the original (low-sensitivity) gFOBT. Also, this FIT requires only 1 stool sample with no dietary restrictions, while both the original gFOBT and the high-sensitivity gFOBT require 3 stool samples and extensive dietary and medication restrictions. Few studies have compared the use of gFOBT to FIT within a broad population, and in observation of a "real world" health care setting.. We evaluated the effectiveness of switching from 3-sample gFOBT testing to 1-sample FIT testing on CRC screening adherence within Kaiser Permanente Northwest (KPNW).

METHODS: We compared adherence to CRC screening in 2 time periods in KPNW: 1) gFOBT era vs. 2) FIT era. The gFOBT era included 69,191 patients between ages 51-80 at average risk for developing CRC and overdue for screening as of July 1, 2008; while the FIT era included similar patients eligible for screening as of July 1, 2009. Patients in both time eras received a 1-minute automated reminder call - encouraging completion of CRC screening - and completion of CRC screening was tracked electronically for 4 months after the completion of the automated call. Screening test adherence was measured as completion of 1 of 4 possible CRC screening tests during the 4-month follow-up period: 1) fecal testing (gFOBT or FIT), 2) colonoscopy, 3) sigmoidoscopy or 4) double contrast barium enema (DCBE). We used logistic regression to analyze the independent effect of fecal test-based time era (gFOBT vs. FIT) on completion of CRC screening, adjusting for age, gender, length of HMO membership, body mass index (BMI), primary care utilization and specialty care utilization.

RESULTS: Patients in the FIT era well more likely to complete CRC screening $(\mathrm{OR}=1.43,95 \% \mathrm{CI}=1.41-1.46)$, compared to those in the gFOBT era. The two populations (FIT era, gFOBT era) were similar with respect to age (FIT mean=60.6, gFOBT mean=60.3), gender (52.8\% female FIT, $52.4 \%$ female), BMI (FIT mean $=30.4$, gFOBT mean $=30.4)$, primary care utilization $(39 \%>1$ visits FIT, $40 \%>1$ visit $\mathrm{gFOBT})$ and specialty care utilization $(53 \%>1$ visits $\mathrm{FIT}, 56 \%>1$ visit gFOBT).

CONCLUSION: Our findings demonstrate that offering a single-sample FIT instead of gFOBT results in increased completion of CRC screening in an integrated care setting. These results suggest that adoption of FIT as a preferred fecal screening test - on a population level - can increase overall adherence to CRC screening. This study is an early step in understanding the effectiveness and cost-effectiveness of FIT testing when utilized in a broad population.

Clinical And Economic Outcomes Among Employees Or Their Dependents With Type-2 Diabetes Initiating Insulin Glargine Via Disposable Pen Or Vial Satish K Garg ${ }^{1}$; Wenhui Wei ${ }^{2}$; Zhun Cao ${ }^{3}$; Stephen N Davis ${ }^{4}$. ${ }^{1}$ Barbara Davis Center for Childhood Diabetes, University of Colorado Denver, Aurora, Colorado ; ${ }^{2}$ Sanofi-Aventis, Bridgewater, New Jersey ; ${ }^{3}$ Thomson Reuters Healthcare, Cambridge, Massachusetts ; ${ }^{4}$ University of Maryland Medical Center, Baltimore, Maryland . (Tracking ID \# 9171)

BACKGROUND: Insulin delivery systems such as pens have the potential to enhance patient acceptance of insulin therapy and therefore tofacilitate diabetes management.

METHODS: This study was designed to examine the difference in clinical and economic outcomes among employees or their dependents 
with type-2 diabetes mellitus (T2DM) initiating insulin glargine via disposable pen or vial. Using the MarketScan commercial claims database from 2007 to 2009, this retrospective cohort study included employees or their dependents aged 18 to 64 yrs with T2DM who were previously on $>=1$ oral medications for diabetes and then initiated insulin glargine via disposable pen or vial. Eligible patients also had to have continuous health plan coverage for $>=6$ months before the initiation date (baseline period) and $>=12$ month afterwards (follow-up period). A 1:1 propensity score match was used to address potential selection bias. Study outcomes included treatment persistence and adherence (as measured by the adjusted medication possession ratio), hypoglycemic events and healthcare resource utilization during the 12 month follow-up period.

RESULTS: A total of 8,136 matched patients $(n=4,068$ in each group) were included in the study. Patients initiating via a disposable pen were significantly more likely to persist $(30.7 \%$ vs. $22 \%, \mathrm{P}<0.0001)$ and adhere $(69.7 \%$ vs. $67.7 \%, \mathrm{P}=0.006)$ to treatment than those initiating insulin glargine via vial during the 12-month follow-up period. Initiation of insulin glargine with the disposable pen was also associated with a reduced risk of hypoglycemia ( $5 \%$ vs. $6.5 \%, \mathrm{P}=0.003$ ), lower likelihood of emergency room visits and hospitalizations, but more visits to endocrinologists (Table 1). Patients in the disposable pen group also had significantly lower total and diabetes-related healthcare costs during the follow-up period (Table 2).

Table 1

\begin{tabular}{|c|c|c|c|}
\hline \multirow[t]{2}{*}{ Outcome measure } & \multirow{2}{*}{$\begin{array}{l}\begin{array}{l}\text { Insulin } \\
\text { glargine } \\
\text { pen users }\end{array} \\
\mathrm{N}=4,068\end{array}$} & \multirow{2}{*}{$\begin{array}{l}\text { Insulin } \\
\text { glargine vial/ } \\
\text { syringe users* } \\
\mathrm{N}=4,068\end{array}$} & \multirow{2}{*}{ 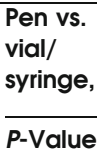 } \\
\hline & & & \\
\hline \multicolumn{4}{|c|}{$\begin{array}{l}\text { All-cause healthcare resource utilization } \\
\text { ED visits }\end{array}$} \\
\hline $\begin{array}{l}\text { Patients with ED visits, } \\
\mathrm{n}(\%)\end{array}$ & $325(8.0)$ & $449(11.0)$ & $<0.001$ \\
\hline $\begin{array}{l}\text { No. of ED visits per } \\
\text { patient, mean } \pm S D\end{array}$ & $0.1 \pm 0.6$ & $0.2 \pm 0.8$ & $<0.001$ \\
\hline \multicolumn{4}{|l|}{ Inpatient admissions } \\
\hline $\begin{array}{l}\text { Patients with inpatient } \\
\text { admission, } \mathrm{n}(\%)\end{array}$ & $568(14.0)$ & $752(18.5)$ & $<0.001$ \\
\hline $\begin{array}{l}\text { No. of inpatient admissions } \\
\text { per patient, mean } \pm \mathrm{SD}\end{array}$ & $0.2 \pm 0.6$ & $0.3 \pm 0.8$ & $<0.001$ \\
\hline $\begin{array}{l}\text { Length of stay per inpatient } \\
\text { admission, mean } \pm \mathrm{SD}\end{array}$ & $5.1 \pm 5.5$ & $5.5 \pm 4.2$ & 0.148 \\
\hline \multicolumn{4}{|l|}{ Visits to endocrinologists } \\
\hline $\begin{array}{l}\text { Patients with visits } \\
\text { to endocrinologists, n (\%) }\end{array}$ & $880(21.6)$ & 753 (18.5) & $<0.001$ \\
\hline $\begin{array}{l}\text { No. of visits } \\
\text { to endocrinologists, } \\
\text { mean } \pm \mathrm{SD}\end{array}$ & $0.7 \pm 1.8$ & $0.6 \pm 1.6$ & 0.001 \\
\hline \multicolumn{4}{|c|}{ Diabetes-related healthcare resource utilization } \\
\hline \multicolumn{4}{|l|}{ ED visits } \\
\hline $\begin{array}{l}\text { Patients with ED visits, } \\
\mathrm{n}(\%)\end{array}$ & $126(3.1)$ & $181(4.4)$ & 0.001 \\
\hline $\begin{array}{l}\text { No. of ED visits per } \\
\text { patient, mean, } \pm \text { SD }\end{array}$ & $0.0 \pm 0.4$ & $0.1 \pm 0.4$ & 0.011 \\
\hline \multicolumn{4}{|l|}{ Inpatient admissions } \\
\hline $\begin{array}{l}\text { Patients with inpatients } \\
\text { admission, } \mathrm{n}(\%)\end{array}$ & $301(7.4)$ & $424(10.4)$ & $<0.001$ \\
\hline $\begin{array}{l}\text { No. of inpatient admissions } \\
\text { per patient, mean } \pm \mathrm{SD}\end{array}$ & $0.1 \pm 0.4$ & $0.2 \pm 0.6$ & $<0.001$ \\
\hline $\begin{array}{l}\text { Length of stay per inpatient } \\
\text { admission, mean } \pm \mathrm{SD}\end{array}$ & $5.0 \pm 3.9$ & $5.3 \pm 3.6$ & 0.182 \\
\hline
\end{tabular}

"Patients were insulin naïve. ED, emergency department; SD, standard deviation
Table 2

\begin{tabular}{|c|c|c|c|}
\hline \multirow[t]{2}{*}{ Outcome measure } & \multirow{2}{*}{$\begin{array}{l}\begin{array}{l}\text { Insulin } \\
\text { glargine } \\
\text { pen users }\end{array} \\
\mathrm{N}=4,068\end{array}$} & \multirow{2}{*}{$\begin{array}{l}\text { Insulin glargine } \\
\text { vial/syringe } \\
\text { users" } \\
\mathrm{N}=4,068\end{array}$} & \multirow{2}{*}{$\begin{array}{l}\begin{array}{l}\text { Pen vs. } \\
\text { vial/ } \\
\text { syringe, }\end{array} \\
P \text {-Value }\end{array}$} \\
\hline & & & \\
\hline \multicolumn{4}{|c|}{ All-cause healthcare cost (\$), mean \pm SD } \\
\hline Outpatient visits & $876 \pm 712$ & $903 \pm 730$ & 0.092 \\
\hline ED visits & $320 \pm 1,172$ & 553 & $<0.001$ \\
\hline Inpatient admissions & 4,090 & 5,90 & $<0.001$ \\
\hline Prescription drugs & 5,70 & 5,3 & 0.011 \\
\hline Total expenditure & $10,991 \pm 21,826$ & 12,61 s & 0.003 \\
\hline \multicolumn{4}{|c|}{ Diabetes-related healthcare cost (\$), mean $\pm \mathrm{SD}$} \\
\hline Outpatient visits & $361 \pm 297$ & $354 \pm 294$ & 0.23 \\
\hline ED visits & $94 \pm 622$ & 128 & 0.016 \\
\hline Inpatient a & $2,050 \pm 11,500$ & 3,109 & 0.001 \\
\hline Prescription drugs & $2,448 \pm 1,612$ & $2,057 \pm 1,537$ & $<0.001$ \\
\hline $\begin{array}{l}\text { Glucose meter and } \\
\text { testing supplies }\end{array}$ & $227 \pm 333$ & $256 \pm 349$ & $<0.001$ \\
\hline Total expenditure & $5,180 \pm 11,643$ & $5,904 \pm 16,946$ & 0.02 \\
\hline
\end{tabular}

"Patients were insulin naïve. ED, emergency department; SD, standard deviation

CONCLUSION: This study suggested that, compared with initiating insulin glargine via a vial, the use of a disposable pen is associated with greater treatment persistence/adherence, decreased hypoglycemia, and potential overall cost savings.

COI: Study funding and editorial support was provided by SanofiAventis, US.

Dissemination and Impact of an Evidence-Based Care Guideline for Patients with Sore Throat in a University Health Center David Dugdale ${ }^{1}$; Mariann Carle ${ }^{2}$. ${ }^{1}$ University of Washington, Seattle, Washington ; ${ }^{2}$ Univeristy of Washington, Seattle, Washington . (Tracking ID \# 10813)

BACKGROUND: Sore throat is a common problem in primary care settings. Our project focused on improving the efficiency and costeffectiveness with which we diagnose and treat patients with sore throat. At baseline, our providers received no specific direction about the most efficient care processes or diagnostic and therapeutic approach. Based on evidence-based practice guidelines, we defined areas for quality improvement including specific measures, and developed, implemented, and assessed a local practice guideline for sore throat.

METHODS: We used encounter diagnosis codes to identify a cohort of patients with possible Group A beta hemolytic strep (GABHS) pharyngitis. We collected data from our Electronic Health Record (EpicCare) for each patient in the cohort including date of index encounter; date of any encounter in the 7 days following the index encounter; age at time of index encounter; and all diagnostic and therapy orders.

RESULTS: We provided care for sore throat to about 350 patients per calendar quarter. The baseline use of throat culture was 55\%, rapid streptococcal antigen test (RSAT) was $70 \%$, and there were 1.30 diagnostic tests per case. After the intervention, $11 \%$ of patients had a GABHS culture $(\mathrm{p}<0.0001), 78 \%$ had a RSAT ( $<<$ 0.001 ), and there were 0.89 diagnostic tests per case. The cost of diagnostic testing fell from $\$ 41$ to $\$ 21$. The rate of antibiotic prescriptions fell from $24 \%$ to $18 \%$ ( $\mathrm{p}<0.0001)$. Among patients prescribed antibiotics, the rate of penicillin prescriptions rose from $50 \%$ to $58 \%(\mathrm{p}<0.01)$.

CONCLUSION: Our guideline process produced significant changes in practice leading to higher quality care at a lower cost. Key components 
of development and implementation were a structured approach, marketed implementation with specific tools in our EHR, and a provider group willing to endorse practice change.

Comparative Effectiveness of Different Point-of-Care Strategies to Improve Antibiotic Use for Acute Bronchitis in Primary Care Ralph Gonzales ${ }^{1}$; Ralph Gonzales ${ }^{1}$; Tammy Anderer ${ }^{2}$; Melissa Stahl ${ }^{3}$; Michelle Yefko ${ }^{3}$; Judith Maselli ${ }^{1}$; Julie Molecavage ${ }^{3}$; Fred Bloom ${ }^{3}$; Joshua Metlay ${ }^{3}$. ${ }^{1}$ UCSF, San Francisco, California ; ${ }^{2}$ Geisinger Health System, Danville, Pennsylvania; ${ }^{3}$ University of Pennsylvania, Philadelphia, Pennsylvania . (Tracking ID \# 11243)

BACKGROUND: The National Committee on Quality Assurance now includes the level of antibiotic prescribing for uncomplicated acute bronchitis $(\mathrm{AB})$ as a HEDIS measure. We have previously shown that providing emergency physicians with an acute cough algorithm based on vital signs and chest examination at the point-of-care was effective at reducing antibiotic prescribing for uncomplicated $\mathrm{AB}$. To evaluate this approach in primary care practices, we partnered with an integrated healthcare system to evaluate two approaches for implementing this algorithm at the point-ofcareâ€"an examination room poster vs. an electronic health record (EHR) standardized intake form and linked order set.

METHODS: In October 2009, we conducted a three-arm, cluster randomized trial across 37 primary care practices. The EHR-based intervention arm (Epic Care) included a management algorithm as a best practice alert linked to a document flow and integrated set of electronic orders. The Poster-based intervention arm included the same management algorithm as an $11 \times 17$ inch laminated poster that was displayed in all examination rooms. Implementation of the algorithms at both types of intervention sites were led by local physician champions. The primary outcome was the proportion of $A B$ visits prescribed an antibiotic within 48 hours of the visit. $A B$ visits were excluded if patients had COPD, immunosuppression or other types of organ failure/ deficiency, or if an antibiotic-responsive secondary diagnosis (such as acute sinusitis, pharyngitis, otitis media or pneumonia) was recorded for the $\mathrm{AB}$ visit. Antibiotic prescription rates for each group in the intervention period (October 2009 â $€$ " March 2010) were compared with similar periods during the prior three years using multi-level adjusted regression models to account for practice and clinician-level clustering. RESULTS: Primary care practices were predominantly family practice (10 of 14 in Control group, 10 of 11 in Poster group and 11 of 12 in EHR group). Baseline patient and physician demographic characteristics did not vary between study groups. Mean and median patient age was 44 years, $60 \%$ of visits were by women. Abnormal vital signs were uncommonly present (3\% to $8 \%$ of visits had elevated temperature, respiratory rate or heart rate). Chest radiography was utilized at the same rate between study groups ( $5 \%$ for all groups). Baseline antibiotic prescription rates for $\mathrm{AB}$ during 3 preceding study periods among Control ( $\mathrm{n}=3433$ visits; $71.8 \%$ ), Poster ( $\mathrm{n}=4020$ visits; $79.5 \%)$ and $\mathrm{EHR}$ sites $(\mathrm{n}=3247$ visits; $74.8 \%)$ varied significantly $(\mathrm{P}<0.001)$. The changes in antibiotic prescription rates during the intervention period at the Poster sites $(n=919 ;-10.6 \%$; P-value $=0.03)$ and the EHR sites $(n=934$; $-14.7 \%$; P-value $=0.05$ ) were significantly different than the change at the control sites $(n=815 ;+0.4 \%)$. There was no difference between the change in antibiotic prescribing at Poster and EHR sites $(\mathrm{P}=0.87)$. The Poster development cost about $\mathbf{\$} 1500$ and the EHR tool development cost about $\$ 18,500$.

CONCLUSION: We conclude that an evidence-based algorithm to guide management of $\mathrm{AB}$ can reduce overuse of antibiotics in primary care settings, but the mode of implementation does not appear to impact the magnitude of effect. Studies of electronic decision support tools that do not compare with more traditional implementation strategies may significantly overestimate the value of electronic decision support.

Health System Capacity and Infrastructure for Adopting Innovations in Venous Thromboembolic Disease Care Danielle Southern ${ }^{1}$; Alka Patel ${ }^{1}$; Nigel Waters ${ }^{2}$; Russell D Hull ${ }^{1}$; Jasmine Poole ${ }^{3}$; Louise Pilote ${ }^{5}$; William Ghali ${ }^{1} .{ }^{1}$ University of Calgary, Calgary, Alberta ; ${ }^{2}$ George Mason University, Fairfax, Virginia ; ${ }^{3}$ McGill University, Montreal, Quebec . (Tracking ID \# 10146)

BACKGROUND: Diagnosis and treatment for venous thromboembolic disease (VTE) has evolved considerably,specifically through diagnostic (e.g., d-dimer testing and better imaging) andtherapeutic innovations (e.g., low molecular weight [LMW] heparins,anticoagulation clinics). Despite the considerable potential for enhancing care, the extent to which these innovations are being adopted in usual care is not known. Furthermore, we recognize that adoption of these innovations requires presence of local infrastructure for their implementation. Recognizing this, we conducted surveys and interviews of health system managers and selected providers within regions and/or hospitals for all of Canada to document the extent of infrastructure for optimal diagnostic care and therapy.

METHODS: We studied system structure for care of VTE disease in 10 provinces and 3 territories and all 94 health regions in Canada. We also documented key elements of system infrastructure at the hospital level for 658 individual acute-care hospitals. We present global Canada-wide data on the proportions of regions or hospitals that have a particular structural attribute along with binomial 95\% confidence intervals for those proportions. We also present detailed tabulations by region and hospital on the presence/absence of each of several specific system structure elements assessed. These included: availability of D-dimers, imaging modalities (CT, ventilation-perfusion (VQ), venography, pulmonary angiography and doppler ultrasonography), low molecular weight heparins on formulary, anticoagulation clinics and local application of critical pathways. We used descriptive statistics and geographic information systems (GIS) to present Canadian health system infrastructure by region and by hospital.

RESULTS: The necessary infrastructure for optimal VTE care was not uniformly available across Canadian hospitals and provinces. Ddimer testing was not available in all hospitals.Larger provinces such as Quebec and Ontario tended to have D-dimers available in most hospitals, whereas smaller ones were more likely to have Ddimer test specimens collected and sent off-site for analysis. Diagnostic imaging modalities such as $\mathrm{CT}$ and ventilation-perfusion scanning were also not uniformly available, and when present, were not always available 24/7. Inter-provincial comparison reveals Quebec to have the greatest availability of diagnostic imaging, whereas Canadaâ $\epsilon^{\mathrm{TM}} \mathrm{s}$ northern territories had minimal availability. Only 6 provinces had regions with dedicated anticoagulation management clinics, or out-patient clinics for the early follow-up of VTE patients. Meanwhile, only Alberta, New Brunswick and Nova Scotia reported having regions with homeanticoagulation (present in $23 \%$ of health regions within these 3 provinces).

CONCLUSION: Health system infrastructure for VTE care in Canada is not entirely in step with the evidence. This research presents an evaluative approach that can be applied in other countries and showcases the relevance of the Donabedian model for health system quality, where optimal system structure is an essential prerequisite to optimized processes and outcomes. The current infrastructure for VTE care in Canada limits the extent to which physicians can provide stateof-the-art care. 
Is Performance of Evidence-Based Processes of Care Associated with Improved Survival for Patients with Community-Acquired Pneumonia? Jonathan S. Lee ${ }^{1}$; Brian A. Primack ${ }^{1}$; Maria K. Mor ${ }^{2}$; Roslyn A. Stone ${ }^{3}$; David S. Obrosky ${ }^{1}$; Donald M. Yealy ${ }^{1}$; Michael J. Fine ${ }^{3}$. ${ }^{1}$ University of Pittsburgh School of Medicine, Pittsburgh, Pennsylvania ; ${ }^{2}$ VA Pittsburgh Healthcare System, Pittsburgh, Pennsylvania ; ${ }^{3}$ Graduate School of Public Health, University of Pittsburgh, Pittsburgh, Pennsylvania . (Tracking ID \# 10238)

BACKGROUND: While often used as proxies for health care quality, performance of processes of care (POCs) may not be associated with improved survival for patients with community-acquired pneumonia (CAP). Our objective was to assess the association between mortality and 4 evidence-based POCs for patients hospitalized with CAP.

METHODS: We studied 2076 patients with clinical and radiographic evidence of CAP directly hospitalized from 32 emergency departments in PA and CT in calendar year 2001. All patients were part of a cluster-randomized trial to assess the effectiveness of guideline implementation in increasing the performance of 4 POCs at presentation (i.e., assessing oxygenation, performing blood cultures, and rapidly initiating and appropriately selecting antibiotic therapy). We followed 2062 (99.3\%) patients for 30 days to assess all-cause mortality. We used hierarchical multiple logistic regression modeling to assess the association between mortality and the individual and total number of POCs performed (i.e., 0-2, 3, or 4), controlling for severity of illness at presentation with the Pneumonia Severity Index and other patient, provider, and site characteristics.

RESULTS: Overall, 2027 (97.6\%) patients had oxygenation assessed, 1314 (63.3\%) had blood cultures performed, and 1632 (78.6\%) received timely (less than 4 hours) and 1308 (63.0\%) received appropriate antibiotic therapy; cumulatively, 534 (25.7\%) patients received 0 to 2,837 (40.3\%) received 3, and $705(34.0 \%)$ received all 4 recommended POCs. Overall, 141 (6.8\%) died. As shown in the Table, mortality was $1.1 \%$ to $1.7 \%$ lower for patients who had each of the individual POCs performed compared to those who did not ( $\mathrm{p}>.15$ for all comparisons); mortality was $7.5 \%$ and $7.2 \%$ for patients that had 0-2 and 3 POCs performed, respectively, compared to $5.8 \%$ for patients that had all 4 POCs performed $(\mathrm{p}=.43)$. Adjusted odds ratios derived from multivariable models showed no statistically significant independent associations ( $\mathrm{p}>.14$ for all comparisons) between mortality and either performance of the individual POCs or the cumulative number of POCs performed.

Associations Between Processes of Care and Mortality for CAP

\begin{tabular}{|c|c|c|c|c|}
\hline \multirow[b]{2}{*}{$\begin{array}{l}\text { Processes } \\
\text { of Care }\end{array}$} & \multicolumn{2}{|c|}{$\begin{array}{l}\text { Mortality (\%) } \\
\text { Performance of } \\
\text { Processes of Care }\end{array}$} & \multirow[b]{2}{*}{ P-Value } & \multirow{2}{*}{$\begin{array}{l}\text { Adjusted } \\
\text { Odds Ratios } \\
\text { for Mortality } \\
(95 \% \mathrm{Cl})\end{array}$} \\
\hline & Performed & $\begin{array}{l}\text { Not } \\
\text { Performed }\end{array}$ & & \\
\hline $\begin{array}{l}\text { Assessment } \\
\text { of oxygenation }\end{array}$ & 6.8 & 8.5 & .65 & $0.7(0.3-1.8)$ \\
\hline $\begin{array}{l}\text { Performance } \\
\text { of blood } \\
\text { cultures }\end{array}$ & 6.7 & 7.0 & .82 & $0.9(0.5-1.6)$ \\
\hline $\begin{array}{l}\text { Antibiotic } \\
\text { administration } \\
(\leq 4 \mathrm{hrs})\end{array}$ & 6.6 & 7.7 & .43 & $0.7(0.5-1.6)$ \\
\hline $\begin{array}{l}\text { Appropriate } \\
\text { antibiotic } \\
\text { selection } \\
\text { Cumulative nur }\end{array}$ & er performe & 7.9 & .15 & $0.8(0.5-1.2)$ \\
\hline $0-2$ & 7.5 & - & .43 & reference \\
\hline 3 & 7.2 & - & & $0.9(0.6-1.3)$ \\
\hline 4 & 5.8 & - & & $0.7(0.4-1.1)$ \\
\hline
\end{tabular}

CONCLUSION: Neither performance of individual POCs nor the cumulative number of POCs performed is associated with short-term survival for patients hospitalized with CAP. Although this study was not originally designed to assess process-outcome associations, our findings challenge the use of POCs as proxy measures of health care quality for CAP.

Obesity and Overweight are Negative Predictors of Colorectal Cancer Screening in a Health Care System Combining Fee for Service and Universal Coverage. Roland Fischer ${ }^{1}$; Tinh-Hai Collet ${ }^{1}$; Andreas Zeller ${ }^{2}$; Lukas Zimmerli ${ }^{3}$; Jean-Michel Gaspoz ${ }^{4}$; Karine Giraudon ${ }^{1}$; Nicolas Rodondi ${ }^{1}$; Jacques Cornuz ${ }^{1} .{ }^{1}$ Department of Ambulatory Care and Community Medicine, University of Lausanne, Lausanne, N/A ; ${ }^{2}$ Medical Outpatient Department / Ambulatory Internal Medicine, University Hospital Basel, Basel, N/A ; ${ }^{3}$ Division of Internal Medicine, University Hospital of Zurich, Zurich, N/A ; ${ }^{4}$ Department of Community Medicine and Primary Care, University Hospitals of Geneva and Faculty of Medicine, Geneva, N/A . (Tracking ID \# 10489)

BACKGROUND: Screening for colorectal cancer (CRC) has been shown to reduce CRC associated mortality by $14 \%$. We aimed to assess predictors of CRC screening in Switzerland, a country with a health care system combining fee forservice and universal coverage.

METHODS: We designed a retrospective cohort study including patients followed in the departments for ambulatory care of university hospitals in Lausanne, Basel, Geneva, and Zurich. A random sample was drawn from administrative data of all patients aged between 50 and 80 years during the review period 2005-2006. Patients with emergency visits, visits to specialized physicians or nurse appointment only, with illegal residency status, and history of CRC or colorectal polyps were excluded. We considered having performed a colonoscopy (CS) in the last 10 years, flexible sigmoidoscopy or double contrast barium enema in the last 5 years, or faecal occult blood testing (FOBT) in the last 2 years as adequate CRCscreening. The patient's refusal of CRC screening $(n=22)$ was also considered as adequate CRC screening, as the preventive care intervention was suggested by the physician. The final sample consisted in 940 patients. As patients could seemore than one physician during the review period, we defined the main physician as the one the patient had the most visits to. Data were abstracted from medical charts and analyzed by multivariate logistical regression using a multilevel generalized linear model. To account for clustering by the 4 sites, we treated each primary care center as a fixed effect. RESULTS: Of the 940, 338 (36\%) patients had an appropriate CRC screening test ( $51 \%$ by CS, $46 \%$ by FOBT, $3 \%$ by sigmoidoscopy). Their mean age was 64 years, $43 \%$ were women, 53\% married, 39\% employed, $40 \%$ retired and $21 \%$ either freelance, unemployed, or under social aid. Most of the patients' consultations (89\%) were performed by residents in general internal medicine rotation at the end of their postgraduate training. 502 patients (55\%) had a female main physician, 64\% a main physician younger than 45 years and the annual median number of visits was 3. Most of the patients had a Charlson index of 3 or lower $(62 \%)$ and a mean Body Mass Index (BMI) of 28.8 kilograms per square meters. Based on the multivariate logistical regression, female gender of the main physician $(\mathrm{OR}=1.59$, 95\% CI $1.17-2.17)$ and having previously received annual influenza vaccination $(\mathrm{OR}=1.48,95 \% \mathrm{CI} 1.02-2.14)$ were significantly associated to CRC screening. BMI between 25.1 and 30 (OR= $0.68,95 \%$ CI 0.47 - 0.98) and between 30.1 and 35 (OR=0.62,95\% CI 0.40 0.97) were negatively associated with CRC screening.

CONCLUSION: Obesity and overweight were negative predictors of CRC screening in the Swiss health care system combining fee for service and universal coverage; female gender of the main physician and annual influenza vaccination were positive predictors. Socioeconomic factor and comorbidity were not significantly associated with CRC screening. 
This implies that physicians' and patients' attitudes towards screening and patient-doctor relationship might be the target of further research and attempts to improve compliance with guidelines on CRC screening.

Osteoporosis in Men with Congestive Heart Failure Tin Kyaw ${ }^{1}$; Bradley Ellis ${ }^{2}$; Sean McFarland ${ }^{3}$; Ronna Mallios ${ }^{4}$; Soe $\overline{\text { Naing }^{2} \text {; Jian }}$ Huang ${ }^{3}$. ${ }^{1}$ VACCHCS (Veterans Affairs Central California Health Care System), Fresno, California ; ${ }^{2} \mathrm{UCSF}$ Fresno department of Internal medicine, Fresno, California ; ${ }^{3}$ VA Central California Health Care System, Fresno, California ; ${ }^{4}$ UCSF Fresno; Grants and Research Office, Fresno, California . (Tracking ID \# 11387)

BACKGROUND: There are limited data regarding osteoporosis among congestive heart failure ( $\mathrm{CHF}$ ) patients. We sought to determine the association between $\mathrm{CHF}$ and the risk of osteoporosis in male veteran patients.

METHODS: A cross sectional chart review was conducted on 1116 male patients who had a dual energy $\mathrm{x}$ ray absorptiometry (DXA) between $12 / 1$ / 2004 and 5/3/2008 at VA Central California Health Care System. The risk factors for osteoporosis including age, weight, smoking and alcohol history, anti-seizure medications, history of levothyroxine use, chronic oral and nasal steroid use, history of chronic obstructive pulmonary disease, hyperthyroidism, hyperparathyroidism, diabetes mellitus, history of thiazolidinedione use, CHF, rheumatoid arthritis, prostate cancer, hormonal treatment for prostate cancer, and chronic kidney disease, were reviewed. Osteoporosis was defined as DXAT score of -2.5 or less in the spine, total hip or femoral neck.

RESULTS: The mean age and weight of the study patients were 69 years (26-94) and $195 \mathrm{Lb}$ (64-429). Out of total 1116 men, 180 were identified to have CHF. The prevalence of osteoporosis in patients with CHF was significantly higher (62 out of 180;34.4\%) than that $(230$ out of $936 ; 24.6 \%)$ in those without $\mathrm{CHF}(\mathrm{P}=0.007)$. In logistic regression analysis, the diagnosis of osteoporosis was significantly associated with the presence of $\mathrm{CHF}$ after adjusting for confounding factors including age, weight, smoking and alcohol status, and above-mentioned co-morbidities and medications (OR:1.516, 95\% CI:1.017-2.260).

CONCLUSION: This study suggests statistically significant association between CHF and increased risk of osteoporosis among our VA male population. This study indicates a need for further research to determine the factors contributing to bone loss among CHF patients.

Physical Examination Teaching: A Systematic Review of the Literature Calvin L. Chou ${ }^{1}$; Somnath Mookherjee ${ }^{1}$; Lara E. Pheatt ${ }^{1} .{ }^{1}$ University of California, San Francisco, San Francisco, California . (Tracking ID \# 7561)

BACKGROUND: Recent reports have bemoaned the inadequacy of the physical examination (PE) skills of physicians, including the "synthesis" of PE data to inform the diagnosis and management of patients. Despite this, most PE curricula in medical education teach a comprehensive $\mathrm{PE}$ without explicit links to application of $\mathrm{PE}$ findings to patient care. Therefore, overall $\mathrm{PE}$ education needs improvement, but the most effective teaching methods are unknown. We performed a systematic review of the literature appraising all studies of $\mathrm{PE}$ curricular interventions to describe teaching methods being used, summarize findings from these studies, and provide guidance for best practices.

METHODS: In collaboration with a research librarian, we systematically searched for all studies of PE teaching published between January 1951 and January 2011. Papers that described a curricular intervention, identified the number of learners, and reported measurements of teaching efficacy were analyzed. Data extracted included the nation of study, level of learner, organ system targeted, study design, types of educational interventions, measurements of efficacy of the intervention, and domains of PE learning targeted for teaching and measurement (including psychomotor skills, knowledge acquisition, communication with examinees, and "synthesis" of collected data into clinical application). At least two authors analyzed each paper.

RESULTS: Of the 3665 papers retrieved, 121 met inclusion criteria. Eighty-seven (72\%) were US studies. Medical students were the primary subjects in $79 \%$ of the included studies, followed by residents (18\%) and practicing physicians (3\%). The most represented organ systems were cardiovascular, musculoskeletal, gynecological, and general (multiple organ systems or comprehensive PE). Sixty-six (55\%) studies included control groups; most other studies used a single group with pre and post measurements or post-only measurements. Major categories of educational interventions included simulation with either standardized patients (SPs) acting as patients or patient-teachers, simulation of aspects of PE with the use of machines (such as recordings of heart sounds or plastic genitourinary models), teaching based on actual patients seen in the hospital or clinic, and peer examination or tutoring. In most studies the intervention included more than one of these methods. Common assessment methods included checklists with observed structured clinical examinations with faculty or SPs as raters to assess psychomotor or communication domains, written knowledge tests, surveys of learner satisfaction and self-assessed competence. Psychomotor skills and knowledge acquisition were the most common competencies targeted; seventeen papers (14\%) were judged to teach PE in the "synthesis" realm.

CONCLUSION: This systematic review found many examples of innovative $\mathrm{PE}$ curricula, with the majority of studies including comparison with a control group. Despite growing recognition that physicians must be competent in applying the PE to make optimal patient care decisions, we found a lack of curricula aiming to teach PE "synthesis" - only 14\% of studies in our analysis targeted this domain. Heterogeneity in the interventions as well as outcome measurements makes it difficult to recommend one curricular intervention over another. We suggest that higher quality studies of PE teaching, particularly in the realm of synthesis, will allow for the identification of best practices in this area.

Regional Variation in Transplant Waitlist: A Contributor to Transplant Disparities Milda R Saunders ${ }^{1}$; Hyo Jung Tak ${ }^{1}$; Lainie Friedman Ross ${ }^{1}$; G. Caleb Alexander ${ }^{1}$. ${ }^{1}$ University of Chicago Medical Center, Chicago, Illinois . (Tracking ID \# 11371)

BACKGROUND: There is substantial regional variation in healthcare quality in the United States. African Americans and Whites are not distributed equally among regions in the US. The objectives of this study were to quantify regional differences in access to the renal transplant waitlist, and to examine how these differences may contribute to racial disparities in waitlist access.

METHODS: Using the United States Renal Data System (USRDS), we examined non-Hispanic Whites $(n=166,874)$ and Blacks $(n=133,474)$ aged 18-70 who initiated dialysis between January 2000 and December 2006. We linked U.S. 2000 Census Data to USRDS data using subjects' zip code at dialysis initiation. We defined our outcome variable as time to transplant waitlist after dialysis initiation. We used the 11 United Network for Organ Sharing (UNOS) regions as indicator variables in order to account for geographic and administrative boundaries. First, 
we used Cox proportional hazards to identify the association between UNOS region and time to transplant waitlist while adjusting for individual (age, gender, insurance and employment status, BMI, and co-morbidities at dialysis initiation) and neighborhood (proportion female headed households, male unemployment, percent poverty and proportion without high school diploma) characteristics. UNOS Region 3 was used as the reference group because it was largest and had longest time to transplant waitlist. Then we used Cox proportional hazards to compare time to transplant waitlist for Whites and African Americans within a given region after adjusting for the individual and neighborhood characteristics above.

RESULTS: The average time to renal transplant waitlisting was 23.7 months and was significantly shorter for Whites (22.1 months) than African Americans (25.8 months, $\mathrm{p}=<0.001)$. Women, those with coronary artery disease, greater neighborhood disadvantage, Medicaid or no insurance at dialysis initiation had a longer average time to waitlisting than their counterparts (all p-values $<0.05$ ). Compared to patients in Region 3 (see Table 1), patients in eight of the ten other regions were more likely to appear on transplant waitlist (adjusted HR 1.1-1.6, p-values<0.05). Compared to Whites within the same region, African Americans had similar times to transplant waitlist in all but three regions. African Americans had a longer time to transplant waitlist than their White counterparts in regions 3 and 4, but waited less time in region 5. Over 30\% of the African American population with end stage renal disease resided in Regions 3 and 4 which have both longer times to transplant waitlist overall and disparities between African Americans and Whites.

Table 1: Transplant Waitlist Outcomes by Region

\begin{tabular}{|c|c|c|c|c|c|}
\hline $\begin{array}{l}\text { UNOS } \\
\text { region }\end{array}$ & States & $\begin{array}{l}\text { Hazard } \\
\text { Ratio (HR) } \\
\text { transplant } \\
\text { waitlist } \\
\text { (p-value) }\end{array}$ & $\begin{array}{l}\text { White- } \\
\text { African } \\
\text { American } \\
\text { HR within } \\
\text { region }\end{array}$ & $\begin{array}{l}\begin{array}{l}\text { Total } \\
\text { population }\end{array} \\
(n=300,348)\end{array}$ & $\begin{array}{l}\text { Proportion } \\
\text { African } \\
\text { American }\end{array}$ \\
\hline 1 & $\begin{array}{l}\mathrm{CT}, \\
\mathrm{VT}, \\
\mathrm{ME}, \\
\mathrm{MA}, \\
\mathrm{NH}, \\
\mathrm{RI}\end{array}$ & $\begin{array}{l}1.86 \\
(<0.01)\end{array}$ & $\begin{array}{l}1.10 \\
(0.14)\end{array}$ & 10630 & \\
\hline
\end{tabular}

CONCLUSION: African Americans are over-represented in geographic regions with longer times to renal transplant waitlist. Within these regions they face "double disadvantage" due to racial disparities in time to transplant waitlist. These regional differences may play an important role in transplant waitlist disparities.

State variation in AIDS Drug Assistance Program prescription drug coverage for modifiable cardiovascular disease risk factors Karen Wang ${ }^{1}$; Oni Blackstock ${ }^{2}$; David Fiellin ${ }^{3}$. ${ }^{1}$ Yale Robert Wood Johnson Foundation Clinical Scholars Program, New Haven, Connecticut ; ${ }^{2}$ VA Connecticut Healthcare System/Yale RWJF Clinical Scholars Program, New York, New York ; ${ }^{3}$ Yale University School of Medicine, New Haven, Connecticut . (Tracking ID \# 10560)

BACKGROUND: Mortality from non-AIDS defining illnesses, such as cardiovascular disease (CVD), has become increasingly common among persons with HIV/AIDS. In the United States, one-third of
HIV-infected persons in care may rely on state-run AIDS Drug Assistance Programs (ADAP) for CVD-related prescription drugs. With no federal requirement regarding ADAP coverage for non-HIV medications, we hypothesized that wide variation exists in state ADAP formularies for the treatment of four modifiable CVD risk factors: type 2 diabetes mellitus (DM), hypertension (HTN), hyperlipidemia, and tobacco use

METHODS: We systematically reviewed 53 state and territorial ADAP formularies to assess the adequacy of drug coverage for DM, HTN, hyperlipidemia, and smoking cessation using evidence-based clinical practice guidelines as the standard of care. ADAPs that covered all first-line drugs for a CVD risk factor were categorized as providing coverage â€œconsistentâ€• with guidelines. ADAPs that covered at least one first-line medication, but not all, for a CVD risk factor were categorized as â€œpartially consistentâ€• ADAPs that provided no first-line medication for a CVD risk factor were categorized as â€œno coverageâ€• .

RESULTS: Of the 53 ADAPs, four (7.5\%) provided consistent coverage for all four CVD risk factors. Thirteen ADAPs $(24.5 \%)$ provided no coverage for all four CVD risk factors. The majority of ADAPs $(68 \%)$ provided at least partially consistent coverage for at least one of the surveyed CVD risk factors. State ADAPs provided coverage consistent with guidelines most frequently for DM $(28 \%)$, followed by HTN (25\%), hyperlipidemia (15\%) and smoking cessation (8\%). Statins $(66 \%)$ were most commonly covered, and nicotine replacement therapies (9\%) were least often covered. Twenty-seven (51\%) state ADAPs did not provide coverage for first-line treatment for DM; $32(60 \%)$ for HTN; 17 (32\%) for hyperlipidemia; and 24 (45\%) for smoking cessation.

CONCLUSION: Consistency of ADAP coverage with guidelines for DM, HTN, hyperlipidemia, and smoking cessation varies widely with many ADAPs providing no coverage for first-line treatment of these conditions. Given the increasing lifespan of HIV-infected persons, that one-third of HIV-infected persons in care rely on ADAPs for prescription coverage, and ADAPsâ€TM restricted budgets, we recommend ADAP prescription drug coverage be consistent with guidelines for CVD risk factors.

The Effect of Clinical Complexity on the Receipt of Comprehensive Care for Diabetes LeChauncy D Woodard ${ }^{1}$; Cassie R Landrum ${ }^{2}$; Degang Wang ${ }^{3}$; Tracy Urech ${ }^{2}$; Salim S Virani ${ }^{3}$; Laura A Petersen ${ }^{3}$. ${ }^{1}$ Michael E. DeBakey VA Medical Center, Houston, Texas ; ${ }^{2}$ Michael E. DeBakey VA Medical Center HSR\&D Center of Excellence, Houston, Texas ; ${ }^{3}$ Michael E. DeBakey VA Medical Center HSR\&D Center of Excellence \& Baylor College of Medicine, Houston, Texas . (Tracking ID \# 10990)

BACKGROUND: Approximately $80 \%$ of patients with diabetes have at least 1 chronic comorbid illness, and $40 \%$ have 3 or more. The importance of comprehensive diabetes care, including blood pressure, lipid, and glycemic control, is widely acknowledged for most diabetic patients. Achieving guideline-recommended treatment goals may have the greatest benefit in preventing diabetes-related complications among the most complex patients, so assessing the magnitude of deficits in care for these patients is important. To examine the relationship between clinical complexity and guideline-recommended care for patients with diabetes, we assessed the impact of comorbidity type, illness burden, and number of comorbidities on achievement of glycemic, blood pressure, and lipid control.

METHODS: We identified 35,872 patients with diabetes receiving primary care between July 2007 and June 2008 at 7 Veterans Affairs 
facilities in 3 states. We assessed quality according to American Diabetes Association guidelines for blood pressure $(<130 / 80 \mathrm{~mm} \mathrm{Hg})$, glycemic (hemoglobin Alc <7\%), and lipid (low-density lipoprotein $<100 \mathrm{mg} / \mathrm{dL}$ ) control. We defined overall good quality of care as the sum of patients meeting a guideline at the time of an index primary care visit and those receiving appropriate care (e.g., appropriate medication added to regimen or follow-up reading controlled) during a 45-day follow-up. We performed 3 separate ordered logistic regression analyses to examine the impact of different measures of clinical complexity on receipt of overall good quality for at least 1 , at least 2 , or all 3 of the diabetes quality measures. We defined clinical complexity by: 1) condition type (diabetes- concordant [related], discordant [unrelated], both, or neither), 2) illness burden (measured by Diagnostic Cost Group Relative Risk Scores), and 3) number of chronic comorbidities. All analyses were adjusted for age and clustering at the facility level.

RESULTS: $718(2.0 \%)$ of patients received overall good quality for 0 of the 3 measures, 3,396 (9.5\%) received overall good quality for only 1 of the 3 measures, 11,262 (31.4\%) received overall good quality for only 2 of the 3 measures, and 20,496 (57.1\%) received overall good quality for all 3 of the measures. When measuring complexity using condition type, patients with both diabetes-concordant and discordant comorbid conditions were more likely than those with no comorbidities to receive overall good quality for all 3 measures (odds ratio [OR], 2.46; 95\% confidence interval $[\mathrm{CI}], 2.24-2.71)$. In addition, patients with the highest illness burden were more likely than those with the lowest illness burden to receive overall good quality for all 3 quality measures (OR 1.62, 95\% CI 1.53-1.71). Further, patients with the highest number of comorbid conditions were more likely than those with no comorbid conditions to receive overall good quality for all 3 quality measures (OR 2.76, 95\% CI 2.51-3.04).

CONCLUSION: We found that over half of diabetic patients received overall good quality for all 3 measures of diabetes care. Patients with the greatest levels of clinical complexity received higher levels of comprehensive care for diabetes compared to less complex patients, regardless of the definition of complexity chosen. While providers may be appropriately targeting the most complex patients for aggressive diabetes management, aiming to improve goal attainment in less complex patients may be an important area for quality improvement.

They Told me to Leave: Women Describe Healthcare Provider Conversations about Intimate Partner Violence Diane $\mathrm{S}$ Morse ${ }^{1}$, Ross Lafleur ${ }^{1}$; Colleen T Fogarty ${ }^{2}$; Mona Mittal ${ }^{3}$; Catherine Cerulli ${ }^{4}$. ${ }^{1}$ University of Rochester School of Medicine, Rochester, New York ; ${ }^{2}$ Department of Family Medicine, Director of Faculty Development Fellowships, Assistant Director of Family Medicine Residency, University of Rochester School of Medicine, Rochester, New York ; ${ }^{3}$ Division of Social and Behavioral Medicine Department of Community and Preventive Medicine, University of Rochester School of Medicine, Rochester, New York ; ${ }^{4}$ Director Laboratory of Interpersonal Violence and Victimization, Department of Psychiatry, University of Rochester, Rochester, New York . (Tracking ID \# 10617)

BACKGROUND: Intimate partner violence (IPV) is a major public health concern affecting $25-50 \%$ of U.S.women in their lifetimes. In primary care, a prevalence of $7-29 \%$ has been reported, with up to $50 \%$ in inner city practices. Approximately $1 / 3$ of women injured during their most recent physical assault received medical treatment, providing an opportunity for health care providers (HCPs) to intervene. Research has demonstrated that primary care clinicians may have difficulty discussing this issue with patients, generating numerous training tools and interventions, to help primary care physicians better address IPV.
We do not know to what extent current primary care practice follows expert recommendations, which include referral to IPV specialists, safety planning, and providing non-judgmental support regardless of the woman's decision to stay or leave the relationship. Physicians may not be knowledgeable regarding risks of leaving the abusive relationship and recommend leaving without adequate safety plans in place, especially for those women who may be at elevated risk for homicide. It is also unknown what degree of trust abused women have in their physicians' advice regarding IPV. The objective of this analysis was to describe a diverse group of female patients' reported experiences talking to their HCPs about their abusive relationships.

METHODS: We conducted structured interviews with 150 women who indicated they experienced IPV in their intimate relationship. We recruited participants from the following settings: family court where women were seeking orders of protection( $(\mathrm{n}=44,29 \%)$, an inner city primary care clinic $(\mathrm{n}=67,45 \%)$, and a hospital-based outpatient psychiatric clinic $(n=39,26 \%)$. We examined 11 questions pertaining to communication with HCPs about IPV, including: "Did your health care provider know about the abuse?", "Which type of provider knew?", "Do you think your health care provider wanted you to stay or leave your violent relationship?" We obtained descriptive statistics for all variables, including respondents' reports about staying or leaving the relationship, trust in HCP, to which HCP they disclosed, and others. We conducted bivariate analyses for the question of leaving or staying in the relationship with the demographic variables and respondent-reported alcohol and drug use. We analyzed the data by recruitment site.

RESULTS: Self-reported demographics were: 47\% (n=71) African American, 37\% $(n=55)$ Caucasian, $7 \%(n=11)$ Hispanic. Participants had low educational attainment and income with $67 \%(\mathrm{n}=100)$ having earned a high school diploma or less and $87 \%(n=131)$ reporting an annual individual income under $\$ 20,000$. Participant age ranged from 18 to over 55 years, with $56 \%(n=84)$ between $26-45$ years, $15 \%(n=22)$ $18-25$ and $14 \%(n=21)$ over 55 . Fifty-one percent $(n=77)$ of participants reported that their HCPs knew of the abuse in their relationship. Of those, $84 \%$ ( $n=65$ total, including $n=25$ obstetrician-gynecologists) of participants reported having told at least one primary care physician. Regarding perceived HCP attitudes, 85\% ( $\mathrm{n}=66)$ reported their HCP was open to talking about IPV, 66\% $(n=51)$ felt comfortableapproaching him/her about it, and $74 \%(n=57)$ felt their HCP was knowledgeable about the topic. Regarding HCP approaches to IPV, among participants whose physician knew of the abuse, $71 \%(n=55)$ reported that their physician wanted them to leave the abusive relationship and $38 \%(n=29)$ stated that they were specifically advised by their physician to leave their abusive partner.Twenty-six percent of the abused women $(n=20)$ reported that they were advised to leave but did not indicate they were given any safety advice. Few participants $(29 \%, n=22)$ reported safety assistance such as referral to community agencies to assist with safety planning. Only 6 women (8\%) stated that physicians offered safety advice and left the decision about leaving or staying in the abusive relationship to the patient. Bivariate analyses found that only age was associated with relevant patient or physician behaviors including reporting IPV to a physician or leaving the abusive relationship, with participants aged 26-35 and 46-55 more likely than the youngest or the oldest groups to report their HCP knew of their IPV, $\mathrm{c} 2(4, \mathrm{~N}=150)=9.79, \mathrm{p}=0.04$.

CONCLUSION: In our study of women recruited from mental health, legal, and primary care settings, participants reported disclosing abuse to primary care physicians almost exclusively, underscoring the importance of the role of primary care physicians in identifying and assisting IPV patients. Most abused women in this sample felt comfortable approaching their physicians about IPV, and believed their physicians to be open to discussing and knowledgeable about IPV. However, in contrast to current physician IPV recommendations to 
remain neutral, most participants $(71 \%)$ felt their physician wanted them to leave their violent relationship. Additionally, most (71\%) were not provided safety advice,which is also in contrast to current guidelines. A positive outcome in this study is the degree of trust that abused women report in their primary care physicians and the extent to which they look to them for help in managing this complex situation. However there is discordance between this trust and the finding that safety planning and a patient-centered approach to the question of staying or leaving the relationship were used rarely by the physicians. These results suggest a need to improve physicians' rates of referral for safety planning and to decrease the focus on leaving the abusive relationship until resources are present to help avoid potentially serious harm.

Urine Sampling in Hospitalised Patients is Erroneous and Unreliable for Clinical Care Surinder Yadav ${ }^{1}$; Mary Ellen-Scales ${ }^{1}$; Mary Brunton ${ }^{1}$; Evan Benjamin ${ }^{1} .{ }^{1}$ Baystate Medical Center, Springfield, Massachusetts . (Tracking ID \# 11211)

BACKGROUND: Hospital acquired infections have taken prominence in the current environment of curtailing inappropriate care. Catheter associated UTI's (CA-UTI) are preventable and high impact secondary to associated costs and poor outcomes. Our UTI prevention group active has worked on prevention and control of CA-UTI, coordinated by infection control, healthcare quality and nursing staff from all medical and surgical units. We have implemented a nursing driven foley removal protocol, focused on prevention guidelines and education around morbidity and mortality associated with CA-UTI. There are no studies that have comprehensively looked at methods of obtaining urine cultures for diagnosis and specimen integrity. Urine culture specimen collection discussions at monthly UTI prevention meetings identified potential gaps in practice which prompted the development of a survey. Our aim was to evaluate standard practice of urine collection and analysis in order to validate improvement processes at reducing CA-UTI.

METHODS: An online and paper survey was provided to dedicated UTI prevention RNs, unit managers and unit staff with expectations to complete the survey. The survey included frontline health care providers responsible for obtaining urine cultures from patients suspect for UTIs. Questions included nationally accepted standardized methods for obtaining specimens, aseptic technique and documentation of specimen type and transport of the sample to the lab.

RESULTS: We had a total of 210 respondents for the survey. Four specimen labeling questions on the survey identified that $72 \%$ of all respondents would not label the specimen correctly; including such as a specimen from a foley catheter, a straight cath, a bagged specimen or the current antibiotic therapy for the patient at the time of urine culture. $9 \%$ of respondents reported they do not complete the technique of obtaining a urine sample in an aseptic manner. 13\% thought that it was acceptable to take a sample from a foley bag and $66 \%$ indicated that they would obtain a sample from the foley bag if it was a newly inserted foley. $19 \%$ claimed that they would obtain a sample from a new bedpan and $4 \%$ said they would obtain a urine culture sample from a cleaned bed pan. For patients who were assessed to be unable to collect a clean catch mid stream urine culture sample, 19\% indicated they would not obtain a straight cath order from a physician, but instead have the patient collect the sample themselves and then send it for testing.

CONCLUSION: Urine sampling seems fraught with possible errors in collection which can influence culture results and patient management. Before assuming that urine culture results are indicative of accurate patient test results, we are retraining and assuring competency of health care providers tasked with this process. Current hospital data may be inaccurate leading to reporting of erroneous infection rates. It is extremely likely that inappropriate urine sampling is leading to misuse and overuse errors with antibiotic treatment of infections that may not exist. Invariably this increases cost of care, exposes patients to unnecessary antibiotic use, and increases risk of multidrug resistant organisms. Every intervention to reduce CA-UTI rates in hospitals will be severely limited because of gaps in practice that exist and unless these are addressed at institutions we will not see a decline.

Use of Pai you Guo, A Pharmaceutically Adulterated Dietary Supplement, among Brazilian-Born Women in the United States Carly Suzanne Benner ${ }^{1}$; Pieter Cohen ${ }^{2}$; Danny McCormick ${ }^{3}$. ${ }^{1}$ Harvard Medical School, Boston, Massachusetts ; ${ }^{2}$ Cambridge Health Alliance, Harvard Medical School, Brookline, Massachusetts ; ${ }^{3}$ Cambridge Health Alliance, Harvard Medical School, Somerville, Massachusetts . (Tracking ID \# 10009)

BACKGROUND: The U.S. Food and Drug Administration (FDA) has identified weight loss dietary supplements that are adulterated with prescription medications and linked to serious adverse events, including deaths. One of these products, Pai You Guo was found to be adulterated with sibutramine and phenolphthalein and recalled by the FDA in November 2009. It is not known if, and to what extent, Pai You Guo is still used, how it is obtained, and what associated side effects it may have.

METHODS: We conducted a survey of Brazilian women who attended a primary care clinic or one of 6 Brazilian churches in an urban community in the Northeastern U.S. to determine the prevalence of Pai You Guo use, common adverse effects, where and how it is obtained, and how the FDA recall has affected its use. Women born in Brazil aged 13 to 60 were recruited to participate from October through December 2010. Data were collected using a self-administered anonymous survey in Portuguese. All eligible women who presented to clinic for an appointment or attended church events at 6 churches were recruited by clinic staff or a study coordinator. Our main outcomes were use of Pai You Gou, and for those indicating use, how the respondent first heard about and obtained the supplement, and side effects experienced during supplement use. For all outcomes, we calculated proportions with $95 \%$ confidence intervals.

RESULTS: Surveys were collected from 542 respondents, 285 from the community (churches) and 257 from clinic patients. The survey was completed by $92.8 \%$ of eligible clinic women and $93.8 \%$ of eligible community women. Forty-seven surveys were excluded because the women were over 60, were not born in Brazil, or did not provide information about their age, country of origin, gender, or use of Pai You Guo, yielding a final sample of 495 surveys (256 from the community and 239 from the clinic). Overall, 120 of the 495 respondents $(24.2 \%$, $95 \%$ CI, 20.5\%, 28\%) reported using Pai You Guo (in tea or pill form) at some time; $22.6 \%$ (95\% CI $17.3 \%, 27.9 \%$ ) of clinic patients and $25.8 \%$ (95\% CI 20.4\%, 31.1\%) of community members used Pai You Guo. Ninety-six of the 120 Pai You Guo users (80\%, 95\% CI 72.8\%, 87.2\%) first heard about Pai You Guo from a friend or family member, and Pai You Guo was most commonly purchased at a store in the U.S. $(64.2 \%$, 95\% CI 55.4\%, 72.6\%). Seventy-one (59.2\%, 95\% CI 50.4\%, 68\%) users reported purchasing Pai You Guo after the FDA recall. Of those who used Pai You Guo, 106 participants (88.3\%, 95\% CI 82.6\%, 94.1\%) experienced side effects. The most common side effects were dry mouth (54.2\%, 95\% CI 45.3\%, 63.1\%), decreased appetite $(50.8 \%, 95 \% \mathrm{CI}$ $41.9 \%, 59.8 \%)$, difficulty sleeping (25.8\%,95\% CI 18\%, 33.7\%), anxiety (25\%, 95\% CI $17.3 \%, 32.8 \%)$, and palpitations $(20 \%, 95 \%$ CI $12.8 \%$, $27.2 \%)$. 
CONCLUSION: This is the first study of the use and mode of procurement of a FDA-recalled pharmaceutically adulterated supplement. We found that its use is prevalent among Brazilian-born women in an urban community in the Northeastern United States and that the vast majority of women experienced side effects. Most women obtained the product from stores inthe U.S. after the FDA recall in November of 2009.

\section{VIGNETTES}

A Case of Prostate Cancer with Unusual Presentation Shunichi Nakagawa ${ }^{1}$; Shahla Baharlou ${ }^{1}$. ${ }^{1}$ Mount Sinai Medical Center, New York, New York . (Tracking ID \# 11530)

LEARNING OBJECTIVES: 1. Diagnose prostate cancer with rare presentation in the elderly patients. 2. Recognize the indication of screening for prostate cancer in healthier older adults.

CASE INFORMATION: Patient is 78 years old male with past medical history of coronary artery disease, atrial fibrillation, hypertension, benign prostate hypertrophy, who presented with near syncope. As baseline, he was a healthy functioning man who was actively teaching at a local college. He had several months' history of back pain and lumbar radiculopathy with radiologic findings of intervertebral disc space narrowing, which had a good response to physical therapy and nonopioid pain control. He decreased food intake intentionally to lose weight in attempt to get more relief and he lost 30 pounds over the previous 3 months before presentation. The day before presentation, he had generalized weakness and dizziness, and almost passed out while standing. He denied any fever, chills, night sweat, nausea, vomiting, or any urinary symptoms. Vital sign was stable and physical examination including rectal examination was unremarkable except he appeared chroniclly ill and had temporal wasting. Initial laboratory showed mildly elevated WBC with neutrophil predominance and isolated elevation of alkaline phosphatase (1780 U/L) and gamma GTP(74 U/L). Echocardiogram showed significant decline (56\% to 35\%) of left ventricular ejection fraction from previous study ( 14 months prior to presentation). He was started on ACE inhibitor without any significant improvement. Subsequently PSA was found elevated to 13 and bone scan showed extensive metastatic disease. He underwent prostate biopsy, which showed prostatic adenocarcinoma with Gleason's score of 9. He was started on androgen deprivation therapy with gonadotropin releasing hormone and bisphosphonate therapy for bone metastasis. Two months later, he developed acute urinary retention caused by outlet obstruction, requiring indwelling urinary catheter. Two weeks after placement of urinary catheter, he was admitted to ICU with urosepsis, requiring intubation and multiple inotoropic agents. He passed away in 2 days with urosepsis caused by Ecoli

IMPLICATIONS/DISCUSSION: Prostate cancer is the most common cancer for male in US. Advances in screening and diagnostic techniques have resulted in detection of prostate cancer at progressively earlier stages and lower levels of prognostic risk. Overall, 68.7\% of cases were diagnosed in the absence of symptoms, including low back pain, hematuria, and/or difficulty with urination (1). This case presented with weight loss and general fatigue. Although malignancy is one of the major differential diagnoses for weight loss, absence of urinary symptoms and normal digital rectal exam made it rather uncommon for prostate cancer to be differential diagnosis.

Regarding the recommendation of prostate cancer screening, at the age of between 40 and 50 depending on the risk, men should start receiving facts about prostate cancer and screening before actually performing screening (2). Screening is by PSA testing every 2-4 years. And screening should be stopped after age 75 years or older or life expectancy less than 10 years (3). In this case, the patient was otherwise medically stable elderly male. It is hard to tell that screening could have detected the disease early enough to have impact on his outcome. Primary care physicians have to continue discussing with their patients about the risk and benefit of screening regularly.

1. Miller, DC, Hafez, KS, Stewart, A, et al. Prostate carcinoma presentation, diagnosis, and staging: an update form the National Cancer Data Base. Cancer 2003; 98:1169.

2. Wolf, AM, Wender, RC, Etzioni, RB, et al. American Cancer Society guideline for the early detection of prostate cancer: update 2010 . CA Cancer J Clin 2010; 60:70

3. U.S. Preventive Services Task Force. Screening for prostate cancer: recommendation and rationale. Ann Intern Med 2002; 137:915

A Young Woman Presenting with Acute Fulminant Hepatic Failure and Severe Hemolysis Ayesha Zahiruddin ${ }^{1}$; Sharon Leung ${ }^{1}$; Darlene LeFrancois ${ }^{2}$. ${ }^{1}$ Montefiore Medical Center, Bronx, New York ; ${ }^{2}$ Monterfiore Medical Center, New York, New York . (Tracking ID \# 11111)

LEARNING OBJECTIVES: 1 . Recognize the key clinical features of Wilsonâ€ ${ }^{\mathrm{TM}} \mathrm{S}$ disease including physical examination findings 2 . Manage the treatment of acute fulminant hepatic failure from Wilsonâ€ $€^{\mathrm{TM}_{\mathbf{S}}}$ disease

CASE INFORMATION: A 22-year-old female presents with 3 days of yellowish discoloration of her eyes and skin. She also noted that her urine was dark and her stools had been a light green color for 3 weeks, accompanied by mild abdominal discomfort. She had never consumed alcohol and took no prescribed or over the counter medications. She had no previous sexual contact and no significant family medical history. Of note, she had been told that she had "abnormal liver function" as per routine blood work done 8 months earlier. She was afebrile with normal hemodynamics. Jaundice with intensely icteric sclera, and mild right upper quadrant tenderness without appreciable hepatomegaly, were notable on exam. She was neurologically intact and stool guaiac was negative. Her initial labs included creatinine 1.5, hematocrit $20 \%$, platelet 135, bilirubin 59, direct bilirubin 34, AST 195, ALT of 27, alkaline phosphatase 14, LDH 1588, albumin 3.1, and INR 3.3. Acetaminophen level was 30.5 (upper normal 30). Ophthalmologic exam with slit-lamp revealed gold-brown deposits at the level of descemetâ $€^{\mathrm{TM}_{\mathrm{S}}}$ membrane. Her serum copper level was elevated at 220. While emergent plasma exchange using fresh frozen plasma replacement was initiated to ameliorate severe intravascular hemolysis and consequent acute renal failure (ARF), she was listed as status 1A for liver transplantation with a presumptive diagnosis of Wilsonâ $€^{\mathrm{TM}_{\mathrm{S}}}$ disease. On day 2 , continuous renal replacement therapy was begun for worsening ARF (creatinine 2.4) which raised the MELD score from 39 to 48. The patient successfully underwent orthotopic liver transplantation on day 4 and was subsequently discharged home on day 18 of hospitalization. Liver histology under rhodanine stain revealed cirrhosis with occasional nodules in which all of the hepatocytes and scattered macrophages contained granular intracytoplasmic copper.

IMPLICATIONS/DISCUSSION: Wilson's disease is a rare autosomal recessive disorder with mutations in the ATP7B gene which impairs the transport of copper from hepatocytes into bile, leading to copper accumulation in tissues including the liver, brain, kidneys, and cornea. The disease was first clinically described in 1912 by K. Wilson as progressive hepato-lenticular degeneration, reflecting that the primary manifestations of the disease relate to copper depositions in liver and lenticular nuclei of the brain. The hepatic presentation is highly 
variable, ranging from asymptomatic to acute fulminant liver failure. Approximately half of patients present without symptoms or with chronic liver disease. These patients typically have low ceruloplasmin levels and frequently (50\%) have pathognomonic Kayser-Fleischer rings on slit lamp exam; they are typically managed as outpatients with chronic chelation and/or zinc therapy. However, fulminant hepatic presentations, more commonly in females than in males (4:1), occur in up to $25 \%$ of cases. Key clinical features include rapidly progressive renal tubular disease, coombs-negative acute intravascular hemolysis, and laboratory discordance manifest as only mildly elevated serum transaminases and alkaline phosphatase but extremely elevated bilirubin levels. Acute hepatocellular necrosis leads to the release of copper ions into the circulation with toxic oxidative effects on red cell metabolic pathways and consequent hemolysis, as well as renal failure. In cases of fulminant hepatic failure, acute plasma exchange is the preferred bridge to liver transplantation since it can rapidly remove relatively large amounts of copper and alleviate hemolysis and further renal damage. Due to the otherwise high mortality and transplant's curative potential, patients with fulminant hepatic failure due to Wilson's disease are appropriately afforded the highest category of priority for liver transplantation.

\section{An Unusual Cause of Deep Vein Thrombosis and Kidney Injury Aries} Caesar Patdu Gavino ${ }^{1}$; Lee $\mathrm{Lu}^{2} .{ }^{1}$ Baylor College of Medicine, Houston, Texas ; ${ }^{2}$ Baylor College of Medicine, Friendswood, Texas . (Tracking ID \# 7329)

LEARNING OBJECTIVES: 1. Recognize retroperitoneal fibrosis as an uncommon cause of deep vein thrombosis and acute kidney injury 2 . Review pathogenesis and treatment of idiopathic retroperitoneal fibrosis CASE INFORMATION: A 49-year-old African American male with no prior medical history presented with 3-months of left upper quadrant pain, left leg swelling, anorexia, weight loss, and decreased urine output. He denied taking any medications and had no prior surgery. Exam revealed lethargy, a soft and non-tender abdomen, and an edematous left lower extremity. Doppler imaging noted a deep venous thrombosis (DVT) in the left leg. He was started on anticoagulation. Admission labs showed creatinine of 20, BUN of 140, and potassium of 7.8, which necessitated hemodialysis. CT of abdomen and pelvis revealed an $8.4 \times 4.8 \times 3.2 \mathrm{~cm}$ retroperitoneal soft tissue density encasing bilateral ureters, infrarenal abdominal aorta, and inferior vena cava. The mass was suspicious for retroperitoneal fibrosis versus lymphoma. MRI showed the same soft tissue growth exhibiting T2 hypointensity consistent with retroperitoneal fibrosis. Further tests included a high CRP at 6.2 and ESR at 111, negative ANA, anti-dsDNA, ANA, and RF. Bilateral nephrostomy tubes were placed, and his kidney function improved. There was no evidence of malignancy. He was diagnosed with idiopathic retroperitoneal fibrosis and was treated with oral prednisone. Six months later, his CRP and ESR returned to normal, and repeat MRI of his abdomen and pelvis showed complete resolution of the retroperitoneal mass.

IMPLICATIONS/DISCUSSION: Retroperitoneal fibrosis is a rare disease with an incidence of 0.1 per 100,000 person-years. It consists of two forms: idiopathic and secondary from medications, surgery, or malignancy. Idiopathic retroperitoneal fibrosis, accounting for $70 \%$ of cases, is seen in individuals 40 to 60 years of age with a sex predominance of 2:1 male to female. Symptoms include flank pain, fever, leg edema, DVT, weight loss, and anorexia. Its pathogenesis involves chronic inflammation and fibroblast proliferation which are thought to be secondary to an autoimmune process. Encasement of the ureters by the mass can cause obstructive uropathy. Obstruction of the venous system leads to venous insufficiency and deep venous thrombosis. CT is the best test to evaluate the extent of fibrosis and to assess lymphadenopathy. The mass is typically confluent and exhibits similar attenuation to that of surrounding muscles. MRI is used to better characterize the mass. Currently, there are no guidelines recommending the need for biopsy. It may be reserved when the mass location is atypical, when the clinical presentation suggests infection or malignancy, or if a patient does not respond to initial therapy. Idiopathic disease is treated with prednisone at $1 \mathrm{mg} / \mathrm{kg}$ daily for 6 weeks and tapered down depending on response. Methotrextae, cyclophosphamide, and mycohenolate are second line drugs. Prognosis is good, and patients usually have resolution of pain, constitutional symptoms, and urinary obstruction with treatment. Recurrences can occur within the first year and are rare. In conclusion, idiopathic retroperitoneal fibrosis should be included in the differential diagnosis in patients presenting with DVT and kidney injury.

Bronchopleural fistula: An Uncommon Complication of Pneumonectomy Caroline Tse ${ }^{1}$; Caroline Tse ${ }^{1}$; Robin Klein ${ }^{1} .{ }^{1}$ Emory University, Atlanta, Georgia . (Tracking ID \# 10628)

LEARNING OBJECTIVES: 1 . Recognize that bronchopleural fistula (BPF) can be a complication of pneumonectomy, which general internists encounter in patients with cancer or severe pulmonaryinfection, in whom pneumonectomy was indicated. 2. Diagnose BPF through key clinical and radiologic findings and manage BPF through treating associated infection, endoscopy, or surgery.

CASE INFORMATION: 43 year-old female presents with productive cough and chest pain. The patient reported a productive cough with whitish sputum associated with pleuritic chest pain for the past 2 months. Her medical history was notable for HIV/AIDS with CD4 count 11 , history of recurrent pneumonias including an episode 1 month ago, and wedge resection of a pulmonary abscess one year prior. On admission, she was febrile with a temperature of $38.0 \hat{A}^{\circ} \mathrm{C}$ but breathing comfortably and not hypoxic. Decreased breath sounds were noted in the left lower lobe along with dullness on percussion. Laboratories revealed a WBC of $4600 / \hat{\mathrm{A}} \mu \mathrm{L}$ and a $\mathrm{PaO} 2$ of $71 \mathrm{mmHg}$. Chest $\mathrm{x}$-ray showed diffuse bilateral fibrotic changes. CT chest noted hydropneumothorax and communication with a basal segmental bronchus. The patient was diagnosed with a bronchopleural fistula (BPF) and recurrent pneumonia. Bronchoscopy was attempted, however the patient did not tolerate this, and the procedure was terminated prior to visualization of the fistula. The hydropneumothorax was drained via pigtail catheter. Operative treatment was not pursued since the fistula appeared to be fibrosing. She was treated for pneumonia with antibiotics.

IMPLICATIONS/DISCUSSION: Bronchopleural fistula (BPF) refers to an anatomical connection between a bronchial airway and pleural space. While uncommon, it is associated with a mortality rate of 16$23 \%$. It is most commonly seen following pneumonectomy although the rate of developing a BPF following pneumonectomy is still low. It may also occur as a complication of infection or radiotherapy. It is more likely to occur after pneumonectomy on the right side than left because of less effective closure of the shorter right bronchus. Preoperative infection, history of radiation, and postoperative positive pressure ventilation increase the risk of this complication in patients following pneumonectomy. BPF may present many years after resection. It is associated with recurrent pneumonia, pneumothorax, effusion, and empyema. CT imaging is highly sensitive, over $90 \%$, for BPF. A key diagnostic feature of BPF is increased air compared to fluid. Normally following pneumonectomy, the space is filled with fluid. In the setting of a BPF, air persists in the pleural space. Imaging may reveal a decrease in the amount of fluid, persistent failure of the 
postpneumonectomy space to fill, mediastinal shift away, subcutaneous or mediastinal emphysema, and pneumothorax. Treatment includes pleural drainage, antibiotics for infection, and closure of the fistula. Small fistulas may be managed conservatively allowing closure via fibrosis while large BPF may require surgical treatment. In conclusion, while uncommon, bronchopulmonary fistula is a serious complication following pneumonectomy, with high morbidity and mortality. Internists should consider BPF in susceptible patients who present with recurrent infections and key radiologic findings.

Celiac Disease Impacts Female Reproduction Sveta Shah ${ }^{1}$; Nisha Basu ${ }^{1}$; Amy Weinstein ${ }^{1} .{ }^{1}$ Beth Israel Deaconess, Boston, Massachusetts . (Tracking ID \# 9265)

LEARNING OBJECTIVES: 1 . Understand the current indications for screening for Celiac Disease (CD). 2. Discuss the evidence for expanding the population currently screened to also include women with unexplained infertility and adverse pregnancy outcomes.

CASE INFORMATION: A 45-year-old G3P1 female with a history of hypertension and iron deficiency anemia attributed to menorrhagia presents to her PCP with fatigue. Review of systems is significant for reflux-type symptoms. She denies abdominal pain, changes in bowel habits, or weight loss. Her other medical history is significant for two second trimester miscarriages and one uncomplicated pregnancy. Her only medication is iron supplements. Family history is significant for colon cancer. Physical exam reveals conjunctival pallor, but is otherwise unremarkable. Laboratory results show worsening anemia, with an initial Hematocrit five years prior of $33 \%$, now $26 \%$. Colonoscopy and upper endoscopy were performed to evaluate for occult bleeding. Duodenal biopsies reveal villous shortening and increased intraepithelial lymphocytes, suggestive of $\mathrm{CD}$. She has since begun a gluten-free diet (GFD) with improvement in her anemia and fatigue.

IMPLICATIONS/DISCUSSION: $\mathrm{CD}$ is an immune-mediated enteropathy triggered by ingestion of gluten, classically associated with gastrointestinal symptoms. In fact, the spectrum of $\mathrm{CD}$ manifestations is actually wider than originally described and particularly pertinent to women of reproductive age. It can manifest with menstrual irregularities, infertility, miscarriage, and intrauterine growth retardation (IUGR). Unfortunately, CD remains vastly undiagnosed, with some demonstrating that only 1 in ten Celiacs are diagnosed. Epidemiologically, $60-70 \%$ of Celiacs are women, so in a disease that remains largely undiagnosed until the fourth decade, many women experience reproductive complications that could potentially be explained earlier with a diagnosis of $\mathrm{CD}$.The data supports only screening targeted populations, rather than mass screening. Indications for screening include chronic gastrointestinal symptoms, unexplained weight loss, iron deficiency anemia, and unexplained vitamin deficiency. However, not only is the prevalence of $\mathrm{CD}$ higher in women with unexplained infertility (up to $4-8 \%$, compared with $1 \%$ prevalence in the general population), but a GFD may lead to improved fecundity following diagnosis. In addition, Celiacs have higher rates of recurrent spontaneous abortions and IUGR, with multiple studies showing a decrease in rate of these complications with adoption of a GFD prior to pregnancy. In keeping with the current practice of case-targeted testing for $\mathrm{CD}$, it seems reasonable to suggest that women with unexplained infertility, unexplained miscarriage, or IUGR be added to the list of indications for screening. In the case above, the patient was diagnosed only towards the conclusion of her reproductive life, which raises the question of whether her previous miscarriages could have been prevented with an earlier diagnosis. Practitioners must maintain a low threshold to test for $\mathrm{CD}$ given possible reversibility of the complications.

Eosinophilia: A Systematic Approach can Lead to an Exciting Diagnosis John N. Mafi ${ }^{1}$; John N. Mafi ${ }^{1} .{ }^{1}$ Beth Israel Deaconess Medical Center, Harvard Medical School, Boston, Massachusetts . (Tracking ID \# 12358)

LEARNING OBJECTIVES: 1. Recognize the common causes of eosinophilia, which are best identified systematically. 2 . Recognize the clinical presentation of adrenal insufficiency, including its association with peripheral eosinophilia.

CASE INFORMATION: A 67 year old woman with a history of ITP, lupus anticoagulant, DVT, returns from her 2 year stay in India to reestablish care with a resident physician complaining of chronic myalgias, arthralgias, and fatigue. Her PMH is otherwise significant for hypothyroidism, and GERD. Her medications are notable for levothyroxine $125 \mathrm{mcg}$ PO daily, and omeprazole $20 \mathrm{mg}$ PO daily. Review of systems is only pertinent for weakness and worsening GERD. Vital signs revealed T 98.0, BP 102/54, HR 80, and RR 16. Physical exam was notable for an elderly, overweight and well-appearing woman. Laboratory analysis were significant for a white count of 11.7 , hematocrit of 38.3 , and platelet count of 39. Differential revealed N 42.9\%, L 30.2\%, and E $21.9 \%$. An initial workup of her eosinophilia yielded 3 negative stool O\&P studies and negative ANA, ANCA, and IgE levels. Her eosinophilia persisted and climbed as high as 3,673 cells/microL.

She was referred to hematology where a bone-marrow biopsy was offered, which she refused. Further workup yielded negative malaria antigen, leishmania, filariasis, histoplasma, strongyloides antibodies, FISH for BCR-ABL, and FIP1L1-PDGFR mutations. Due to worsening GERD and pruritis, concern was raised for hypereosinophilic syndrome complicated by eosinophilic esophagitis. She was referred for an endoscopy that yielded multiple negative biopsies. Finally, she was referred to ID to expand the infectious workup given her stay in India. Her ID physician raised the possibility of chronic adrenal insufficiency, after skillfully eliciting a history of long term steroid use for ITP in India. This diagnosis was subsequently supported by a random cortisol of 4.1 $\mathrm{mcg} / \mathrm{dL}$. After starting prednisone $5 \mathrm{mg}$ PO daily, the diagnosis was further solidified by the patient's claim of improved weakness, arthralgias, myalgias, "greater energy levels" and a normalized absolute eosinophil count of 140 cells $/$ microL.

IMPLICATIONS/DISCUSSION: According to the great detective Sherolock Holmes, "when you have eliminated the impossible, whatever remains, however improbable, must be the truth." In our case, an extensive work up for our patient's eosinophilia ruled out multiple possibilities and allowed the process of elimination lead to diagnosis. Despite this complex work up, it is important to recall that the first step in diagnosing eosinophilia lies in a basic history and physical exam and calculating an absolute count of $>500$ cells/ micrroL. Eosinophilia most commonly reflects an allergic, infectious, or neoplastic process. Important clues consist of recent travel suggesting an infectious cause, which should prompt a regionspecific workup; and new medications or rhinitis, which both point towards a hypersensitivity etiology. Considering broad categories based on pathophysiology avoids missing key conditions. These categories include allergic (atopy and medications), infectious (typically parasitic), hematologic (lymphoproliferative diseases), specific organ-related diseases (ie eosinophilic pneumonia), immune dysfunction (autoimmune disease), vascular disorders (cholesterol emboli), and endocrinologic (adrenal insufficiency). 
The pathophysiologic basis for eosinophilia in adrenal insufficiency lies in cortisol's role in inducing apoptosis of the eosinophil. The most common symptoms include weakness, fatigue, anorexia, and GI complaints and less commonly musculoskeletal complaints, most of which were present in our patient. The most common signs consist of weight loss, hyperpigmentation, hypotension, and electrolyte disturbances. The diagnosis is confirmed by a morning cortisol of $<3 \mathrm{mcg} / \mathrm{dL}$, while a morning level of $<10 \mathrm{mcg} / \mathrm{dL}$ suggests the diagnosis. Pharmacologic treatment centers on glucocorticoid replacement. Laboratory values should not guide dosage, which instead should be guided by symptom relief and maintained at the lowest dose possible.

\section{Is a Necrotic Foot a Terminal Illness in a 92 Year Old Frail Man?} Hillary Lum ${ }^{1}$; Hollis Day ${ }^{1} .{ }^{1}$ University of Pittsburgh, Pittsburgh, Pennsylvania . (Tracking ID \# 10512)

LEARNING OBJECTIVES: 1. Apply appropriate prognostic tools to complex medical issues affecting frail older adults. 2. Recognize the need for guiding in-the-moment decision making.

CASE INFORMATION: Mr. $\mathrm{W}$ is a 92 year old Holocaust survivor with a history of coronary artery disease, stroke, and peripheral vascular disease, who was admitted to the hospital with a 3 week history of progressive pain, swelling and redness of his foot, associated with a $4 \mathrm{~cm}$ necrotic ulcer. His symptoms had limited his ability to walk for days. Review of systems included $10 \mathrm{lb}$. weight loss, decreased intake, trouble swallowing, a non-productive cough, and a sacral pressure ulcer. He lived with his wife in assisted living and received $4 \mathrm{hrs}$ /day of paid care-giver assistance. He needed help with all activities of daily living (ADL). His daughter, who was his health care power of attorney (POA), stated his cognition was "slipping a bit". Prior to admission, his goals of care, as discussed by his POA with his primary MD, were no resuscitation or intubation, and to avoid nursing home care due to the POA's concern for emotional distress related to his prior imprisonment. Upon admission, i.v. antibiotics were started and an MRI revealed osteonecrosis with associated abscess and cellulitis. Laboratory studies showed WBC 16,900, hgb $12.0 \mathrm{~g} / \mathrm{dL}$, creatinine $1.2 \mathrm{mg} / \mathrm{dL}$, and albumin $3.0 \mathrm{~g} / \mathrm{dL}$. A vascular surgery consultant recommended "urgent, non-elective" surgical debridement, prolonged antibiotics, and a definitive procedure if the first procedure was tolerated. Despite the patient's progressive decline in functional status, recent weight loss and productive cough, concerning for malnutrition, aspiration pneumonia, and limited wound healing, his POA wanted to consider possible interventions for his acute gangrene. Potential risks of the procedure included post-operative delirium, hospital-acquired infections, pain, nursing home placement, and multiple rehospitalizations. The key clinical dilemma was whether proceeding with surgical debridement and possible amputation would result in medical care, especially nursing facility placement, that were contrary to his goals of care.

IMPLICATIONS/DISCUSSION: The question of when to offer an invasive medical procedure to a frail elderly patient with an acute illness, recognizing the balance between previously stated goals of care, the desire to intervene, and prognosis with or without surgical intervention, highlights two challenges: 1) Determining and discussing prognosis remains difficult. Population-based prognostic indices are not specific enough. Based on functional status and comorbidities, the patient's 1-year mortality was more than $68 \%(1)$, without considering acute medical issues or outcomes based on management. Few studies compare outcomes of surgical or medical management in frail elderly patients. A recent study of geriatric patients showed that measures of frailty, such as limited mobility and ADL dependence, predicted postsurgical adverse outcomes, including discharge to an institution, after elective surgery(2). Still, physicians have trouble determining or are unwilling to recognize when an event may be terminal, thus limiting their ability to make recommendations. 2) Advance directives are often not specific enough to guide in-the-moment decision making. Acute events require complex decisions based on the clinical context, shifting and evolving goals, and patients' and surrogates' needs(3). Primary physicians must serve as advocates by synthesizing these factors, providing specific recommendations, and helping patients and surrogates choose from available options. The physician must balance patient autonomy with a broad understanding of the risks of specific procedures, including prolonged hospitalization, for the older frail patient. In summary, primary physicians caring for frail elderly patients need to appropriately incorporate prognostic indices that include markers of frailty in on-going in-the-moment decision making with patients and families. 1. Walter et al. JAMA. 2001;285(23):2987-94. 2. Makary et al. J Am Coll Surg. 2010;210(6):901-8. 3. Sudore et al. Ann Int Med. 2010;153(4):256-61.

One Way Ticket From the Bladder to the Heart Daniel Vogel ${ }^{1}$; Sabrina Jegerlehner ${ }^{1}$; Drahomir Aujesky ${ }^{1} .{ }^{1}$ University Hospital Bern, Bern, N/A . (Tracking ID \# 11001)

LEARNING OBJECTIVES: 1. Recognize Escherichia coli as the prinzipal non-HACEK gram-negativ bacillus causing infective endocarditis 2. Assessment of gram-negativ bacteremia should include infective endocarditis, especially in patients with endovascular devices or preexisting valvular heart disease

CASE INFORMATION: A 76 year old female was admitted to our emergency ward approximatly one month after a 10 day antibiotic treatment with amoxicillin/clavulanic acid because of Escherichia coli septicemia following an urinary tract infection. 10 days prior to admittance she had become increasingly adynamic and developed daily episodes of fever up to 39 degrees celcius and chills. Clinical examination revealed a febrile and tachypneic patient. Laboratory findings were remarkable for a white blood cell count of 18,7 G/1 with a $31 \%$ left shift, toxic granulations and a CRP value of $187 \mathrm{mg} / \mathrm{L}$. Chest $\mathrm{x}$-ray and urinary tests revealed no focus of infection. Four blood cultures were drawn, all showing growth of Escherichia coli.

Particularly because our patient had a history of mitral valve reconstruction two years earlier, transesophageal echocardiography was performed. It revealed a vegetation on the mitral valve with abscess formation on the anterior and perforation of the posterior leaflet (Fig.1). In accordance with the modified DUKE criteria infective endocarditis was diagnosed and antibiotic treatment with ceftriaxone and gentamicin was started. Control echocardiography six days later showed disease progression with meanwhile severe mitral stenosis due to a floating vegetation measuring $25 \times 12 \mathrm{~mm}$ (Fig. 2). Therefore immediate mitral valve replacement was performed.

Eubacterial PCR of the removed valve and the abscess material was positive and identified 16S-rDNA consistent with Escherichia coli.

IMPLICATIONS/DISCUSSION: Infective endocarditis caused by nonHACEK (species other than Haemophilus, Actinobacillus actinomycetemcomitans, Cardiobacterium hominis, Eikenella corrodens or Kingella) gram-negative bacilli is very rare, accounting for less than $2 \%$ of reported cases. Among these, Escherichia coli is the principal pathogen. Less frequent organisms reported are Pseudomonas aeruginosa, Klebsiella and Serratia species. Presumed source of infection often involves the genitourinary tract. The mitral valve is most commonly affected and implanted 
endovascular devices are frequently involved. The clinical course tends to be severe; the in-hospital mortality and complication rates are high, despite high rates of subsequent cardiac surgery

Previously associated with IV drug use, infective endocarditis caused by non-HACEK gram-negative bacilli has recently revealed to be a nosocomial problem. Although rare, it is clinically significant given the number of patients at risk, the great increase in use of endovascular devices which have been shown to be a common risk factor, the growing problem of antibiotic resistence in gram-negative bacteria and the high associated mortality rate.

Some data suggest that elderly patients with predisposing cardiac diseases and Escherichia coli urinary tract infection/bacteremia should generally be examined for endocarditis.

Unusual Case of Dyspnea Alpa Vora ${ }^{1}$; Vinod Khatri ${ }^{2}$; Ayesha Salahuddin ${ }^{3}$; Sangmesh Jabshetty ${ }^{2}{ }^{1}$ St. Francis hospital Illinois, Evanston, Illinois ; ${ }^{2}$ St. Francis Hospital, Evanston, Illinois ; ${ }^{3}$ Saint Francis hospital, Evanston, Illinois . (Tracking ID \# 12030)

LEARNING OBJECTIVES: 1. Recognize Ogilvie's syndrome as a cause of abdominal distension and dyspnea.

2. Diagnose and treat a case of Ogilvie's syndrome early to avoid life threatening complications.

CASE INFORMATION: 64 yrs old male was admitted with history of cough, chest congestion and shortness of breath for 5 days and abdominal pain for 1 day. His past medical history included Diabetes, Hypertension, CAD, CVA with right sided weakness, Seizure disorder, Major depression, Hypercholesterolemia. In ER, patient was alert, in mild distress and vital signs revealed temp- 98.8 F, P-103/min, BP103/89 mm Hg, RR- 24/min, O2 saturation- 97\% on room air. Exam: Neck: No JVD, Chest: reduced air entry on Rt side with high pitched bowel sounds heard in right lower chest. Cardiac: S1S2 N, no murmur, Abdomen: soft, slightly distended abdomen with tenderness (Rt $>$ Lt), high pitched bowel sounds. Extremities: slight weakness of right compared to left. All labs including electrolytes, Cardiac markers, Amylase, lipase were normal, EKG- normal, Chest Xray: large dilated air-filled loop of bowel occupying the lower two-thirds of the right hemithorax, possible eventration or diaphragmatic hernia. CT Abdomen: normal small bowel loops, markedly dilated proximal colon which may be compatible with Ogilvie syndrome, no evidence of bowel obstruction or volvulus. Patient was managed conservatively with trial of Metoclopramide and Erythromycin with no relief. Patient developed increasing shortness of breath and underwent decompressive Colonoscopy and later on Subtotal Colectomy with Ileostomy.

IMPLICATIONS/DISCUSSION: Ogilvie's syndrome is most commonly reported in patients in the sixth decade, and is predominant in men. It is characterized by clinical and radiological evidence of acute large bowel obstruction in the absence of a mechanical cause. The pathophysiology of Ogilvie syndrome is not clearly understood. An imbalance in the autonomic innervation appears to lead to a functional bowel obstruction, as supported by pharmacologic and spinal blockade studies, metabolic abnormalities, and retroperitoneal trauma. Common associations include trauma, infection, cardiac (MI, heart failure), neurological (Parkinson's, spinal cord injury, multiple sclerosis), major orthopaedic surgery, electrolyte imbalance, drugs (Opiods, Calcium channel blockers, phenothiazines, epidural analgesics, alpha-2 agonists). It is associated with significant morbidity and mortality, and, therefore, requires urgent gastroenterologic evaluation. Increasing age, cecal diameter, delay in decompression, and status of the bowel significantly influence mortality, which is approximately $40 \%$ when ischemia or perforation is present. The risk of colonic perforation in ACPO increases when cecal diameter exceeds $12 \mathrm{~cm}$ and when the distention has been present for greater than 6 days. Appropriate management includes supportive therapy and selective use of neostigmine and colonoscopy for decompression. Early recognition and management are critical in minimising complications. Neostigmine is an effective treatment with response rates of $60-90 \%$ and requires cardiovascular monitoring.

\section{IME}

Challenges in Teaching Quality Improvement Skills to Third Year Medical Students: Feasibility and Lessons Learned Somnath Mookherjee ${ }^{1}$; David Levitt ${ }^{1} .{ }^{1}$ University of California, San Francisco, San Francisco, California . (Tracking ID \# 11870)

SETTING AND PARTICIPANTS: Sixteen students in a UCSF third year longitudinal integrated clerkship were eligible to participate; half were assigned to this curriculum.

DESCRIPTION: Developmentally appropriate QI competencies were identified using an accepted framework. Students were required to identify a quality gap based on their interests or clinical experiences, describe existing efforts to address the gap, make preliminary quantifying measures and make a final presentation proposing an intervention to close the quality gap. Presentations were evaluated with a validated tool for assessing trainee QI proposals (QIPAT-7). Knowledge and attitude surveys were administered at the beginning and end of the curriculum. Reaction to the curriculum was assessed through surveys and a focus group.

NEEDS AND OBJECTIVES: Systems based practice and practice based learning are key competencies that medical trainees are expected to learn at all levels. Training in quality improvement (QI) has thus emerged as a major focus in medical education. Due to differences in clinical experience and knowledge, trainees at different levels require curricula in $\mathrm{BI}$ tailored to their developmental stage. We developed a year long experience-based QI curriculum targeting third year medical students.

EVALUATION: All eight students completed the year-long curriculum. Working in two groups of four, students successfully identified quality gaps, stakeholders affected by the gap, and identified means to measure whether the gap would narrow as a result of their proposed interventions. Although knowledge about key QI concepts did not change over time, students' attitudes demonstrated a trend towards higher valuation of QI by the end of the curriculum. Finding appropriate projectspecific mentorship was difficult for one group, ("...it was very frustrating to try ... to have to find our own mentor"). Assessment of the final projects demonstrated weakness with higher order tasks, but students appreciated the opportunity to share their work, ("Great experience to put [our work] together into a finished product").

DISCUSSION/REFLECTION/LESSONS LEARNED: A year-long QI curriculum for medical students is feasible, and may improve students' appreciation of the importance of QI. Future efforts to teach QI at this level of training may benefit from increased dedicated didactics to teach key concepts rather than rely on experience based learning, as well as prioritization of early establishment of project-specific mentorship.

Teaching Clinical Reasoning and Evidence-Based Physical Examination at the Bedside: A Pilot Study Somnath Mookherjee ${ }^{1}$; Calvin L Chou ${ }^{1}$. ${ }^{1}$ University of California, San Francisco, San Francisco, California . (Tracking ID \# 11868)

SETTING AND PARTICIPANTS: Twenty-one senior medical students enrolled in Advanced Physical Examination elective. 
DESCRIPTION: The elective includes an introduction to the principles of EBPE. We developed a three hour structured bedside rounding session to reinforce key EBPE skills: estimation of pretest probability of the disease, recognition of findings, evidencebased estimation of post-test probability of the disease, and decision making based on this assessment. For example, for a patient with dyspnea, elevated neck veins, S3, and abdominojugular reflux, students decide whether to diurese the patient or order an echocardiogram. Session structure:

- Two to four patients examined at the bedside.

- Examination technique and findings verified.

- Students commit to the next best management step.

- Faculty provide feedback on decision making.

Students answered questionnaires that measured confidence in their ability to perform EBPE, perceived utility of $\mathrm{PE}$, and usefulness of elective activities. Clinical vignettes presenting practice choices were used to assess clinical reasoning.

NEEDS AND OBJECTIVES: The evidence-based physical examination (EBPE) is an important diagnostic tool; however, there are few studies that apply EBPE to patient management. Generally, medical school PE education is based on skill acquisition in three domains: psychomotor (motor skills), affective (interaction with examinees), and cognitive (interpretation). Recent studies have emphasized a "synthetic" domain: physicians must recognize findings, understand their evidence-based utility, and then make optimal management decisions. To improve students' ability to synthetically use EBPE in clinical practice, we modified a fourthyear medical student elective in the physical examination to include structured bedside rounds.

EVALUATION: Three 2 week electives were offered between November 2009 and March 2010. Twenty-one students participated. Using a Likert scale ( $1=$ not at all confident, $5=$ very confident), students showed increased confidence in (a) their PE skills in general (retrospective pre: $3.38+/-0.50$, post: $4.19+/-0.40, \mathrm{p}<0.05$ ), (b) understanding significance of findings (retrospective pre: $2.86+/-$ 0.57 , post: $4.23+/-0.44, \mathrm{p}<0.05$ ) and (c) making management decisions based on findings (retrospective pre: $2.90+/-0.87$, post: $4.57+/-0.60, \mathrm{p}<0.05)$. "Bedside CHF rounds" was the highest rated activity for improving $\mathrm{PE}$ skills. Most students requested that the elective include more bedside rounds. Vignettes to assess clinical reasoning had high pre-elective scores and did not change appreciably in post-elective testing.

DISCUSSION/REFLECTION/LESSONS LEARNED: Our pilot of structured bedside rounds to teach senior medical students to recognize findings, understand their evidence-based utility, and use assessments to make optimal management decisions was well received and valued by students. Students reported increased confidence in making management decision based on PE findings at the end of the elective. However, improvement in clinical reasoning could not be detected using our assessments, indicating that better calibrated instruments to measure the "synthetic" domain must be developed.
IPM

Patient Navigator Assistance with Screening Mammography in an Urban Resident Primary Care Practice Vasudev Mandyam ${ }^{1}$; Renee McKinney ${ }^{1}$; Bonnie Sherman ${ }^{1}$; Karen M. Freund ${ }^{1}$. ${ }^{1}$ Boston Medical Center, Boston, Massachusetts . (Tracking ID \# 10718)

STATEMENT OF PROBLEM OR QUESTION: Screening mammography rates in our resident clinics are below our institutional target; do patient navigators help increase these rates in an underserved population?

DESCRIPTION OF PROGRAM/INTERVENTION: A senior medical resident introduced other trainees to the PN model as part of a quality improvement seminar. The navigator's role and collaboration between navigator and resident were discussed. Feedback was positive, with residents reporting high interest in this initiative.

We selected a clinic site in which 24 residents practice, spanning all three years of training.We provided them with practice data identifying the current mammography status of their female patients. Residents met individually with the navigator to review these lists.Women age 50-70 were screened for enrollment in the navigation protocol if they were near-overdue for mammography, defined as having their last mammogram over 18 months ago. Under resident guidance, a navigator provided intensive outpatient case management to assist in mammogram completion. Residents learned to provide clinical decision-making while the navigator focused on outreach and tracking. OBJECTIVES OF PROGRAM/INTERVENTION:

Implement patient navigators (PN) to assist with screening mammography. PNs scheduled and tracked tests to completion, and alerted providers to abnormal results.

Measure the impact of PN on screening rates, aiming to reach the $80 \%$ HEDIS benchmark and achieve diagnostic resolution of abnormal tests. Assess the impact of PN on primary care education. Given the trend towards medical home care models, we focused on teaching housestaff to be team leaders.

FINDINGS TO DATE: There were a total of 157 females age 50-70 in the resident panels, with an average of 6.5 patients per resident. Of these, 58 were overdue ( $>24$ months), yielding a $63 \%$ screening rate. This is well below both the HEDIS benchmark of $80 \%$ and the $78 \%$ rate in faculty practice panels. We also identified 6 women with past screening abnormalities and incomplete follow up. A total of 73 patients were nearoverdue ( $>18$ months). Of these, 69 were referred by their physician for navigation. After 2 months of navigation, preliminary results reveal 19\% have completed mammography, $20 \%$ are in the process of completing their care, 26\% are undergoing navigator tracking, and 33\% did not receive mammography, with the most common reasons being inability to contact $(n=11)$, refusal $(n=5)$, or transfer of care $(n=8)$. Of the 6 women with prior abnormalities, one has had a normal repeat study, one is unreachable, one transferred care, and three are being tracked.

KEY LESSONS LEARNED: We identified an important gap between expected and actual rates of breast cancer screening among resident practice panels. We implemented the PN model to address this gap. The data-gathering process is ongoing and robust. We learned that residents are interested in quality improvement and learning to work in teams to provide patient care. 